\title{
Article
}

\section{Transport Efficiency of Continuous-Time Quantum Walks on Graphs}

\author{
Luca Razzoli ${ }^{1, *(D)}$, Matteo G. A. Paris ${ }^{2,3}$ (D) and Paolo Bordone $1,4, *$ (D) \\ 1 Dipartimento di Scienze Fisiche, Informatiche e Matematiche, Università di Modena e Reggio Emilia, \\ I-41125 Modena, Italy \\ 2 Quantum Technology Lab, Dipartimento di Fisica Aldo Pontremoli, Università Degli Studi di Milano, \\ I-20133 Milano, Italy; matteo.paris@fisica.unimi.it \\ 3 INFN, Sezione di Milano, I-20133 Milano, Italy \\ 4 Centro S3, CNR-Istituto di Nanoscienze, I-41125 Modena, Italy \\ * Correspondence: luca.razzoli@unimore.it (L.R.); paolo.bordone@unimore.it (P.B.)
}

Citation: Razzoli, L.; Paris, M.G.A.; Bordone, P. Transport Efficiency of Continuous-Time Quantum Walks on Graphs. Entropy 2021, 23, 85. https://doi.org/10.3390/e23010085

Received: 27 November 2020

Accepted: 7 January 2021

Published: 9 January 2021

Publisher's Note: MDPI stays neutral with regard to jurisdictional clai$\mathrm{ms}$ in published maps and institutional affiliations.

Copyright: (C) 2021 by the authors. Licensee MDPI, Basel, Switzerland. This article is an open access article distributed under the terms and conditions of the Creative Commons Attribution (CC BY) license (https:// creativecommons.org/licenses/by/ $4.0 /)$.

\begin{abstract}
Continuous-time quantum walk describes the propagation of a quantum particle (or an excitation) evolving continuously in time on a graph. As such, it provides a natural framework for modeling transport processes, e.g., in light-harvesting systems. In particular, the transport properties strongly depend on the initial state and specific features of the graph under investigation. In this paper, we address the role of graph topology, and investigate the transport properties of graphs with different regularity, symmetry, and connectivity. We neglect disorder and decoherence, and assume a single trap vertex that is accountable for the loss processes. In particular, for each graph, we analytically determine the subspace of states having maximum transport efficiency. Our results provide a set of benchmarks for environment-assisted quantum transport, and suggest that connectivity is a poor indicator for transport efficiency. Indeed, we observe some specific correlations between transport efficiency and connectivity for certain graphs, but, in general, they are uncorrelated.
\end{abstract}

Keywords: transport on graph; quantum walk; transport efficiency; connectivity

\section{Introduction}

A continuous-time quantum walk (CTQW) is the quantum mechanical counterpart of the continuous-time random walk. It describes the dynamics of a quantum particle that continuously evolves in time in a discrete space, e.g., on the vertices of a graph, obeying the Schrödinger equation [1,2]. The Hamiltonian describing a CTQW is usually the Laplacian matrix $L$, which encodes the topology of the graph and it plays the role of the kinetic energy of the walker. Experimentally [3], CTQWs can be implemented on nuclearmagnetic-resonance quantum computers [4], optical lattices of ultracold Rydberg atoms [5], quantum processors [6], and photonic chips [7]. The applications of CTQWs range from implementing fast and efficient quantum algorithms [8,9], e.g., for spatial search [10] and image segmentation [11], to implementing quantum logic gates by multi-particle CTQWs in one-dimension (1D) [12], from universal computation [13] to modeling and simulating quantum phenomena, e.g., state transfer [14-16], quantum transport, and for characterizing the behavior of many-body systems [17,18].

Indeed, modeling quantum transport processes by means of CTQWs is a well-established practice and an appropriate mathematical framework. Quantum transport has been investigated with this approach on restricted geometries [19], semi-regular spidernet graphs [20], Sierpinski fractals [21], and on large-scale sparse regular networks [22]. CTQWs have been used in order to model transport of nonclassical light in coupled waveguides [23], coherent exciton transport on hierarchical systems [24], small-world networks [25], Apollonian networks [26], and on an extended star graph [27], coherent 
transport on complex networks [28], and exciton transfer with trapping [29,30]. It is worth noting that CTQWs do not necessarily perform better than their classical counterparts, since the transport properties strongly depend on the graph, the initial state, and on the propagation direction under investigation [31]. A measure of the efficiency of quantum and classical transport on graphs by means of the density of states has been proposed in [32].

Biological systems are known to show quantum effects [33,34] and efficient transport processes. Hence, the great interest in also studying CTWQs to model, e.g., exciton transport on dendrimers [35], photosynthetic energy transfer [36], environment-assisted quantum transport [37], dephasing-assisted transport on quantum networks and biomolecules [38], excitation transfer in light-harvesting systems [39,40], and its limits [41]. There also studies concerning disorder-assisted quantum transport on hypercubes and binary trees [42], because the latter can model a dendrimer-like structure for artificial light-harvesting systems $[43,44]$.

Therefore, a full characterization of the transport properties on different structures is desired. Formally speaking, the CTQW Hamiltonian modeling transport processes shows similarities with the CTQW Hamiltonian adopted to study the spatial search. Both of them consist of the sum, with proper coefficients, of the Laplacian matrix, which is accountable for the motion of the walker on the graph, and the projector onto one or more specific vertices. This projector is the trapping Hamiltonian in transport problems and the oracle Hamiltonian in spatial search problems. The regularity, global symmetry, and connectivity of the graph have proved to be unnecessary for fast spatial search [45-47] by invoking certain graphs, e.g., complete bipartite graphs, strongly regular graphs, joined complete graphs, and a simplex of complete graphs, as counterexamples of these false beliefs. In this work, we address the transport by CTQW on the above mentioned graphs, which are different in terms of regularity, symmetry, and connectivity, and we assess the transport efficiency for initial states that are localized at a vertex and for an initial superposition of two vertices. Our focus is on the role of connectivity, if any. Indeed, regularity and global symmetry are not required for efficient transport, because removing some edges in the complete graph and the hypercube, which are regular and highly symmetric graphs, has been shown to improve the transport efficiency [48].

The paper is organized, as follows. In Section 2, we introduce CTQWs on a graph. In Section 3, we review the dimensionality reduction method to analyze CTQW problems [48], according to which we obtain a reduced model of the Hamiltonian encoding the problem that is considered and the reduced Hamiltonian still fully describes the dynamics that are relevant to the problem. In Section 4, we define the Hamiltonian modeling the transport on graphs and the transport efficiency as a figure of merit to measure the transport properties of the system. For each graph considered, we provide the reduced Hamiltonian and compute the transport efficiency for different initial states. In Section 5, we assess different measures of connectivity in order to characterize each graph considered. Finally, we present our conclusions in Section 6. In Appendix A, we report and refine the proof of the equality of the two subspaces that are required for computing the transport efficiency. In Appendix B, we determine the basis states spanning such a subspace for each graph considered.

\section{Continuous-Time Quantum Walks}

A graph is a pair $G=(V, E)$, where $V$ denotes the non-empty set of vertices and $E$ the set of edges. The order of the graph is the number of vertices, $|V|=N$. We define the adjacency matrix

$$
A_{j k}= \begin{cases}1 & \text { if }(j, k) \in E, \\ 0 & \text { otherwise }\end{cases}
$$

which describes the connectivity of $G$, and $D$ the diagonal degree matrix with $D_{j j}=\operatorname{deg}(j)$, the degree of vertex $j$. In terms of these matrices, we introduce the graph Laplacian $L=D-A$, which is the matrix representation of the graph. According to this definition, $L$ is positive semidefinite and singular. 
The CTQW is the propagation of a quantum particle with kinetic energy when confined to a discrete space, e.g., a graph. The CTQW on a graph $G$ takes place on a $N$-dimensional Hilbert space $\mathcal{H}=\operatorname{span}(\{|v\rangle \mid v \in V\})$, and the kinetic energy term $(\hbar=1) T=-\nabla^{2} / 2 m$ is replaced by $T=\gamma L$, where $\gamma \in \mathbb{R}^{+}$is the hopping amplitude of the walk. The state of the walker obeys the Schrödinger equation

$$
i \frac{d}{d t}|\psi(t)\rangle=H|\psi(t)\rangle,
$$

with Hamiltonian $H=\gamma L$. Hence, a walker starting in the state $\left|\psi_{0}\right\rangle \in \mathcal{H}$ continuously evolves in time, according to

$$
|\psi(t)\rangle=U(t)\left|\psi_{0}\right\rangle,
$$

with $U(t)=\exp [-i H t]$ the unitary time-evolution operator. The probability to find the walker in a target vertex $w$ is therefore $\left|\left\langle w|\exp [-i H t]| \psi_{0}\right\rangle\right|^{2}$.

\section{Dimensionality Reduction Method}

In most CTQW problems, the quantity of interest is the probability amplitude at a certain vertex of the graph. The graph encoding the problem to solve often contains symmetries that allow for us to simplify the problem, since the evolution of the system actually occurs in a subspace of the complete $N$-dimensional Hilbert space $\mathcal{H}$ that is spanned by the vertices of the graph. We can determine the minimal subspace that contains the vertex of interest and it is invariant under the unitary time evolution via the dimensionality reduction method for CTQW, as proposed by Novo et al. [48], which we briefly review in this section for completeness. Such a subspace, also known as a Krylov subspace [49], contains the vertex of interest and all powers of the Hamiltonian applied to it. The relevance and the power of this method is that the graph encoding a given problem can be mapped onto an equivalent weighted graph, whose order is lower than the order of the original graph and whose vertices are the basis states of the invariant subspace. The corresponding reduced Hamiltonian still fully describes the dynamics that are relevant to the considered problem.

The unitary evolution (3) can be expressed as

$$
|\psi(t)\rangle=\sum_{k=0}^{\infty} \frac{(-i t)^{k}}{k !} H^{k}\left|\psi_{0}\right\rangle
$$

so $|\psi(t)\rangle$ is contained in the subspace $\mathcal{I}\left(H,\left|\psi_{0}\right\rangle\right)=\operatorname{span}\left(\left\{H^{k}\left|\psi_{0}\right\rangle \mid k \in \mathbb{N}_{0}\right\}\right)$. This subspace of $\mathcal{H}$ is invariant under the action of the Hamiltonian and, thus, also of the unitary evolution. Naturally, $\operatorname{dim} \mathcal{I}\left(H,\left|\psi_{0}\right\rangle\right) \leq \operatorname{dim} \mathcal{H}=N$, but, if the Hamiltonian is highly symmetrical, only a small number of powers of $H^{k}\left|\psi_{0}\right\rangle$ are linearly independent, so the dimension of $\mathcal{I}\left(H,\left|\psi_{0}\right\rangle\right)$ can be much smaller than $N$.

Let $P$ be the projector onto $\mathcal{I}\left(H,\left|\psi_{0}\right\rangle\right)$, so we have that

$$
U(t)\left|\psi_{0}\right\rangle=P U(t) P\left|\psi_{0}\right\rangle=\sum_{k=0}^{\infty} \frac{(-i t)^{k}}{k !}(P H P)^{k}\left|\psi_{0}\right\rangle=e^{-i P H P t}\left|\psi_{0}\right\rangle=e^{-i H_{\mathrm{red}} t}\left|\psi_{0}\right\rangle,
$$

where $H_{\text {red }}=P H P$ is the reduced Hamiltonian, and we used the fact that $P^{2}=P$ (projector), $P\left|\psi_{0}\right\rangle=\left|\psi_{0}\right\rangle$, and $P U(t)\left|\psi_{0}\right\rangle=U(t)\left|\psi_{0}\right\rangle$.

For any state $|\phi\rangle \in \mathcal{H}$, which we consider to be the solution of the CTQW problem, we have

$$
\left\langle\phi|U(t)| \psi_{0}\right\rangle=\left\langle\phi|P P U(t) P| \psi_{0}\right\rangle=\left\langle\phi\left|P e^{-i H_{\text {red }} t}\right| \psi_{0}\right\rangle=\left\langle\phi_{\text {red }}\left|e^{-i H_{\text {red }} t}\right| \psi_{0}\right\rangle,
$$


where, the reduced state, $\left|\phi_{\text {red }}\right\rangle=P|\phi\rangle$. Reasoning analogously with the projector $P^{\prime}$ onto the subspace $\mathcal{I}(H,|\phi\rangle)$, we obtain

$$
\left\langle\phi|U(t)| \psi_{0}\right\rangle=\left\langle\phi\left|e^{-i H_{\text {red }}^{\prime}}\right| \psi_{0 \text { red }}\right\rangle,
$$

with $H_{\text {red }}^{\prime}=P^{\prime} H P^{\prime}$ and $\left|\psi_{0 \text { red }}\right\rangle=P^{\prime}\left|\psi_{0}\right\rangle$.

An orthonormal basis of $\mathcal{I}(H,|\phi\rangle)$, as denoted by $\left\{\left|e_{1}\right\rangle, \ldots,\left|e_{m}\right\rangle\right\}$, can be iteratively obtained, as follows: the first basis state is $\left|e_{1}\right\rangle=|\phi\rangle$, then the successive ones are obtained by applying $H$ on the current basis state and orthonormalizing with respect to the previous basis states. The procedure stops when we find the minimum $m$ such that $H\left|e_{m}\right\rangle \in$ $\operatorname{span}\left(\left\{\left|e_{1}\right\rangle, \ldots,\left|e_{m}\right\rangle\right\}\right)$. The reduced Hamiltonian, i.e., $H$ written in the basis of the invariant subspace, has a tridiagonal form, so the original problem is mapped onto an equivalent problem that is governed by a tight-binding Hamiltonian of a line with $m$ sites.

\section{Quantum Transport}

The CTQW on a graph $G(V, E)$ of $N$ vertices provides a useful framework to model, e.g., the dynamics of a particle or a quasi-particle (excitation) in a network. The quantum walker moves under the Hamiltonian

$$
H=\gamma L=\gamma \sum_{i \in V} \operatorname{deg}(i)|i\rangle\langle i|-\gamma \sum_{(i, j) \in E}(|i\rangle\langle j|+| j\rangle\langle i|),
$$

which can be read as a tight-binding Hamiltonian with uniform nearest-neighbor couplings $\gamma$ and on-site energies $\gamma \operatorname{deg}(i)$. In the following, we set the units such that $\gamma=\hbar=1$, so hereafter time and energy will be dimensionless.

However, in general, an excitation does not stay forever in the system in which it was created. In biological light-harvesting systems, the excitation gets absorbed at the reaction center, where it is transformed into chemical energy. In such a scenario, the total probability of finding the excitation within the network is not conserved. We assume a graph in which the walker can only vanish at one vertex $w \in V$, known as trap vertex or trap. The component of the walker's wave function at the trap vertex is absorbed by the latter at a trapping rate $\kappa \in \mathbb{R}^{+}$[28]. Therefore, to phenomenologically model such loss processes we have to change the Hamiltonian (8), so we introduce the trapping Hamiltonian

$$
H_{\text {trap }}=-i \kappa|w\rangle\langle w|,
$$

which is anti-hermitian. This leads to the desired non-unitary dynamics that are described by the total Hamiltonian

$$
H=L-i \kappa|w\rangle\langle w|
$$

This Hamiltonian has the same structure as the Hamiltonian for the spatial search of a marked vertex $w$ [10], i.e., it is the sum of the Laplacian matrix and the projector onto $|w\rangle$, with proper coefficients. For spatial search, the projector onto $|w\rangle$ plays the role of the oracle Hamiltonian and the search Hamiltonian is hermitian. For quantum transport, the projector onto $|w\rangle$, because of the pure imaginary constant, plays the role of the trapping Hamiltonian (9) and the transport Hamiltonian (10) is not hermitian.

The transport efficiency is a relevant measure for a quantum transport process [37], which can be defined as the integrated probability of trapping at the vertex $w$

$$
\eta=2 \kappa \int_{0}^{+\infty}\langle w|\rho(t)| w\rangle d t=1-\operatorname{Tr}\left[\lim _{t \rightarrow+\infty} \rho(t)\right],
$$

where $2 \kappa\langle w|\rho(t)| w\rangle d t$ is the probability that the walker is successfully absorbed at the trap within the time interval $[t, t+d t]$ and $\rho(t)=|\psi(t)\rangle\langle\psi(t)|$ is the density matrix of the walker. The second equality of Equation (11) is due to the following reason. The surviving total probability of finding the walker within the graph at time $t$ is $\langle\psi(t) \mid \psi(t)\rangle=\operatorname{Tr}[\rho(t)]$ 
and it is $\leq 1$ because of the loss processes at the trap vertex. Because the transport efficiency is the integrated probability of trapping in the limit of infinite time, we can also assess the transport efficiency as the complement to 1 of the probability of surviving within the graph, which is the complementary event.

In this scenario, there is no disorder in the couplings or site energies of the Hamiltonian or decoherence during the transport. In this ideal regime computing the transport efficiency amounts to finding the overlap of the initial state with the subspace $\Lambda(H,|w\rangle)$ spanned by the eigenstates of the Hamiltonian $\left|\lambda_{k}\right\rangle$ having a non-zero overlap with the trap $|w\rangle$, as proved by Caruso et al. [40]. Indeed, the dynamics are such that the component of the initial state within the space $\Lambda$ is absorbed by the trap, whereas the component outside this subspace, i.e., in $\bar{\Lambda}=\mathcal{H} \backslash \Lambda$, remains in the graph (see Figure 1 ). Let us expand the initial state on the basis of the eigenstates of the Hamiltonian

$$
\left|\psi_{0}\right\rangle=\sum_{k=1}^{m}\left\langle\lambda_{k} \mid \psi_{0}\right\rangle\left|\lambda_{k}\right\rangle+\sum_{k=m+1}^{N}\left\langle\lambda_{k} \mid \psi_{0}\right\rangle\left|\lambda_{k}\right\rangle=\left|\psi_{\Lambda}\right\rangle+\left|\psi_{\bar{\Lambda}}\right\rangle,
$$

where we assume the eigenstates form an orthonormal basis (in the case of degenerate energy levels, we consider the eigenstates after orthonormalization) and are ordered in such a way that $\Lambda=\operatorname{span}\left(\left\{\left|\lambda_{k}\right\rangle \mid 1 \leq k \leq m\right\}\right)$ and $\bar{\Lambda}=\operatorname{span}\left(\left\{\left|\lambda_{k}\right\rangle \mid m+1 \leq k \leq N\right\}\right)$. The components in $\bar{\Lambda}$ are not affected by the open-dynamics that act at the trap vertex $w$. The remaining components evolve in the subspace $\Lambda$ that is defined by having a finite overlap with the trap and are therefore absorbed at the trap. In the limit of $t \rightarrow+\infty$ the net result is the following: the total survival probability of finding the walker in the graph is $\left\langle\psi_{\bar{\Lambda}} \mid \psi_{\bar{\Lambda}}\right\rangle \leq 1$, i.e., it is due to the part of the initial state expansion in $\bar{\Lambda}$; instead, the part of the initial state expansion in $\Lambda$ is fully absorbed at the trap, and so $\eta=\left\langle\psi_{\Lambda} \mid \psi_{\Lambda}\right\rangle=\sum_{k=1}^{m}\left|\left\langle\lambda_{k} \mid \psi_{0}\right\rangle\right|^{2}$. A further consequence of this is that, if the system is initially prepared in a state $\left|\psi_{0}\right\rangle \in \bar{\Lambda}$, then the walker will stay forever in the graph without reaching the trap $(\eta=0)$; if the system is initially prepared in a state $\left|\psi_{0}\right\rangle \in \Lambda$, then the walker will be completely absorbed by the trap $(\eta=1)$.

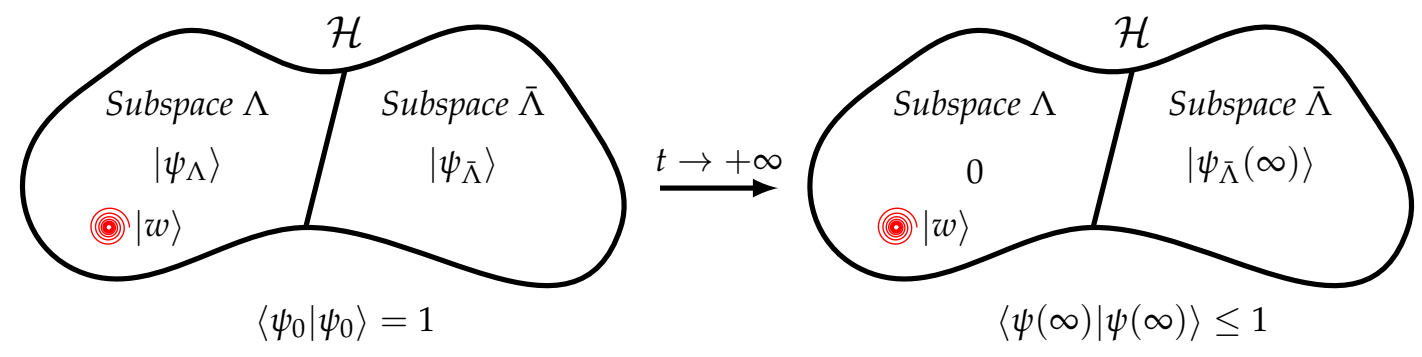

Figure 1. The quantum walker is in the initial state $\left|\psi_{0}\right\rangle(12)$ and it has components in $\Lambda(H,|w\rangle)$, the subspace spanned by the eigenstates of the Hamiltonian having a non-zero overlap with the trap $|w\rangle$, and in $\bar{\Lambda}=\mathcal{H} \backslash \Lambda$, the complement of $\Lambda$ in the complete Hilbert space $\mathcal{H}$. In the limit of $t \rightarrow+\infty$, the dynamics are such that the component having non-zero overlap with the trap is fully absorbed by the trap, i.e., $\left|\psi_{\bar{\Lambda}}(\infty)\right\rangle=0$, whereas the component in $\bar{\Lambda}$ survives. The dynamics are not unitary and the total survival probability of finding the walker within the graph is not conserved, i.e., $\langle\psi(\infty) \mid \psi(\infty)\rangle \leq 1$.

If, on the one hand, this analytical technique allows for one to compute the transport efficiency without solving dynamical equations, on the other hand diagonalizing the Hamiltonian might still be a hard task. The dimensionality reduction method in Section 3 allows for one to avoid diagonalizing the Hamiltonian, since it can be proved that $\Lambda(H,|w\rangle)=\mathcal{I}(H,|w\rangle)$ (see Appendix A). Hence, we compute the transport efficiency as

$$
\eta=\sum_{k=1}^{m}\left|\left\langle e_{k} \mid \psi_{0}\right\rangle\right|^{2}
$$


i.e., as the overlap of the initial state $\left|\psi_{0}\right\rangle$ with the subspace $\mathcal{I}(H,|w\rangle)=\operatorname{span}\left(\left\{\left|e_{k}\right\rangle \mid 1 \leq\right.\right.$ $k \leq m\})$.

We consider as the initial state either a state localized at a vertex, $\left|\psi_{0}\right\rangle=|v\rangle$, or a superposition of two vertices, $\left|\psi_{0}\right\rangle=\left(\left|v_{1}\right\rangle+e^{i \theta}\left|v_{2}\right\rangle\right) / \sqrt{2}$. The localized initial state is a paradigmatic choice to take into account the fact that an excitation is usually created locally in a system. We also considered a superposition in order to investigate possible effects of coherence. The transport efficiency for the superposition of two vertices

$$
\eta_{s}=\frac{1}{2} \sum_{k=1}^{m}\left|\left\langle e_{k} \mid v_{1}\right\rangle+e^{i \theta}\left\langle e_{k} \mid v_{2}\right\rangle\right|^{2}
$$

can be easily assessed, in some cases, when knowing the transport efficiency $\eta_{1}$ and $\eta_{2}$ for an initial state localized at $v_{1}$ and $v_{2}$, respectively. If $\left|v_{1}\right\rangle$ and $\left|v_{2}\right\rangle$ have the same overlap with the basis states, i.e., $\left\langle e_{k} \mid v_{1}\right\rangle=\left\langle e_{k} \mid v_{2}\right\rangle$ for $1 \leq k \leq m$, then $\eta_{1}=\eta_{2}=\eta$, and we have

$$
\eta_{s}(\theta)=\frac{1}{2}\left|1+e^{i \theta}\right|^{2} \eta=(1+\cos \theta) \eta,
$$

so $0 \leq \eta_{s}(\theta) \leq 2 \eta$. Instead, if $\left|v_{1}\right\rangle$ and $\left|v_{2}\right\rangle$ have nonzero overlap with different basis states, i.e., $\left\langle e_{k} \mid v_{1}\right\rangle \neq 0$ for $1 \leq k \leq m_{1}$ and $\left\langle e_{k} \mid v_{2}\right\rangle \neq 0$ for $m_{1}+1 \leq k \leq m_{2}$, with $m_{2} \leq m$, then we have

$$
\eta_{s}=\frac{1}{2}\left(\eta_{1}+\eta_{2}\right)
$$

and it is does not depend on $\theta$.

In the following sections, we study quantum transport on different graphs that are relevant in terms of symmetry, regularity, and connectivity. For each graph, we determine the basis of the subspace in which the system evolves, the reduced Hamiltonian (10), and the transport efficiency (13) for an initial state localized at a vertex or a superposition of two vertices that is not covered by Equation (15). To analytically deal with a graph, we will group together the vertices that identically evolve by symmetry $[45-47,50]$. We mean that such vertices behave identically under the action of the Hamiltonian, in the sense that they are equivalent upon the relabeling of vertices, as well as, e.g., all of the vertices in a complete graph are equivalent. This does not mean that the time evolution $\left|v_{1}(t)\right\rangle$ of an initial state localized at a vertex $v_{1}$ is exactly equal to the time evolution $\left|v_{2}(t)\right\rangle$ of another initial state localized at $v_{2} \neq v_{1}$, but it means that these two time evolutions are the same upon exchanging the labels of the two vertices. Note that the Hamiltonian (10) acts on a generic vertex as the Laplacian, except for the trap vertex, which, thus, forms a subset of one element, itself. The equal superpositions of the vertices in each subset form a orthonormal basis for a subspace of the Hilbert space and the Hamiltonian written in such a basis still fully describes the evolution of the system. However, we point out that such basis spans a subspace which, in general, is not the subspace $\mathcal{I}(H,|w\rangle)$ we need to compute the transport efficiency. Nevertheless, this grouping of vertices provides a useful framework to analytically deal with the system and, for this reason, we will introduce it. Clearly, identically evolving vertices have the same transport properties. However, vertices that are not equivalent for the Hamiltonian can provide the same transport efficiency. For this reason, in the following, we will stress when this is the case.

\subsection{Complete Bipartite Graph}

The complete bipartite graph (CBG) $G\left(V_{1}, V_{2}, E\right)$ is a highly symmetrical structure, which, in general, is not regular. The CBG has two sets of vertices, $V_{1}$ and $V_{2}$, such that each vertex of $V_{1}$ is only connected to all of the vertices of $V_{2}$ and vice versa. The set of CBGs is usually denoted as $K_{N_{1}, N_{2}}$, where the orders of the two partitions $N_{1}=\left|V_{1}\right|$ and $N_{2}=\left|V_{2}\right|$ are such that $N_{1}+N_{2}=N$, with $N$ the total number of vertices. The CBG is non-regular as long as $N_{1} \neq N_{2}$ (see $K_{4,3}$ in Figure 2), and the star graph is a particular case of CBG with $N_{1}=N-1$ and $N_{2}=1$. Without a loss of generality, we assume the trap vertex $w \in V_{1}$. 


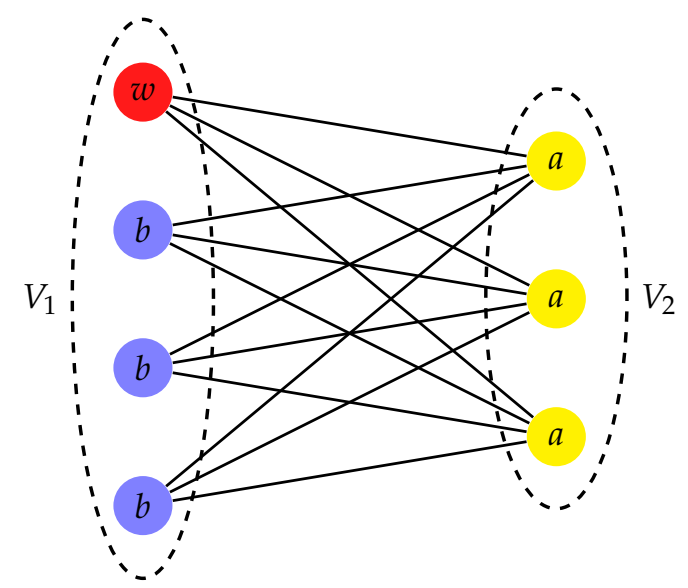

Figure 2. Complete bipartite graph $K_{4,3}$. The trap vertex $w \in V_{1}$ is colored red. Identically evolving vertices have the same transport properties and are identically colored and labeled.

The system evolves in a 3-dimensional subspace (see Appendix B.1) that is spanned by the orthonormal basis states

$$
\left|e_{1}\right\rangle=|w\rangle, \quad\left|e_{2}\right\rangle=\frac{1}{\sqrt{N_{2}}} \sum_{i \in V_{2}}|i\rangle, \quad\left|e_{3}\right\rangle=\frac{1}{\sqrt{N_{1}-1}} \sum_{\substack{i \in V_{1}, i \neq w}}|i\rangle .
$$

This is also the basis that we would obtain by grouping together the identically evolving vertices in the subsets $V_{a}=V_{2}$ and $V_{b}=V_{1} \backslash\{w\}$ (see Figure 2) [45]. In this subspace, the reduced Hamiltonian is

$$
H=\left(\begin{array}{ccc}
(1-\alpha) N-i \kappa & -\sqrt{(1-\alpha) N} & 0 \\
-\sqrt{(1-\alpha) N} & \alpha N & -\sqrt{(1-\alpha)(\alpha N-1) N} \\
0 & -\sqrt{(1-\alpha)(\alpha N-1) N} & (1-\alpha) N
\end{array}\right),
$$

where $\alpha=N_{1} / N \in \mathbb{Q}^{+}, N_{2}=(1-\alpha) N$, since $N_{1}+N_{2}=N$. Notice that, for $G$ to be a CBG, $\alpha$ must satisfy the condition $1 / N \leq \alpha \leq 1-1 / N$.

If the initial state is localized at a vertex $v \neq w$, then the transport efficiency is

$$
\eta= \begin{cases}\frac{1}{\alpha N-1} & \text { if } v \in V_{1} \\ \frac{1}{(1-\alpha) N} & \text { if } v \in V_{2}\end{cases}
$$

and we observe that

$$
\eta_{1}<\eta_{2} \quad \Leftrightarrow \quad 2 \alpha>1+\frac{1}{N},
$$

where $\eta_{1(2)}:=\eta\left(v \in V_{1(2)}\right)$. Instead, if the initial state is a superposition of two vertices, each of which belongs to a different partition, i.e., $v_{1} \in V_{1} \backslash\{w\}$ and $v_{2} \in V_{2}$, then the transport efficiency

$$
\eta_{s}=\frac{N-1}{2 N(\alpha N-1)(1-\alpha)}
$$

follows from Equation (16), so clearly $\eta_{2(1)} \leq \eta_{s} \leq \eta_{1(2)}$, where the alternative depends on the condition (20). The transport efficiency depends on the parameters of the graph, $N$ and $\alpha$, as well as on the initial state (see Figure 3). Whether we consider an initial localized state or a superposition of two localized states, the asymptotic behavior is $\eta=O(1 / N)$ if $N_{1}$ and $N_{2}$ are both sufficiently large. 

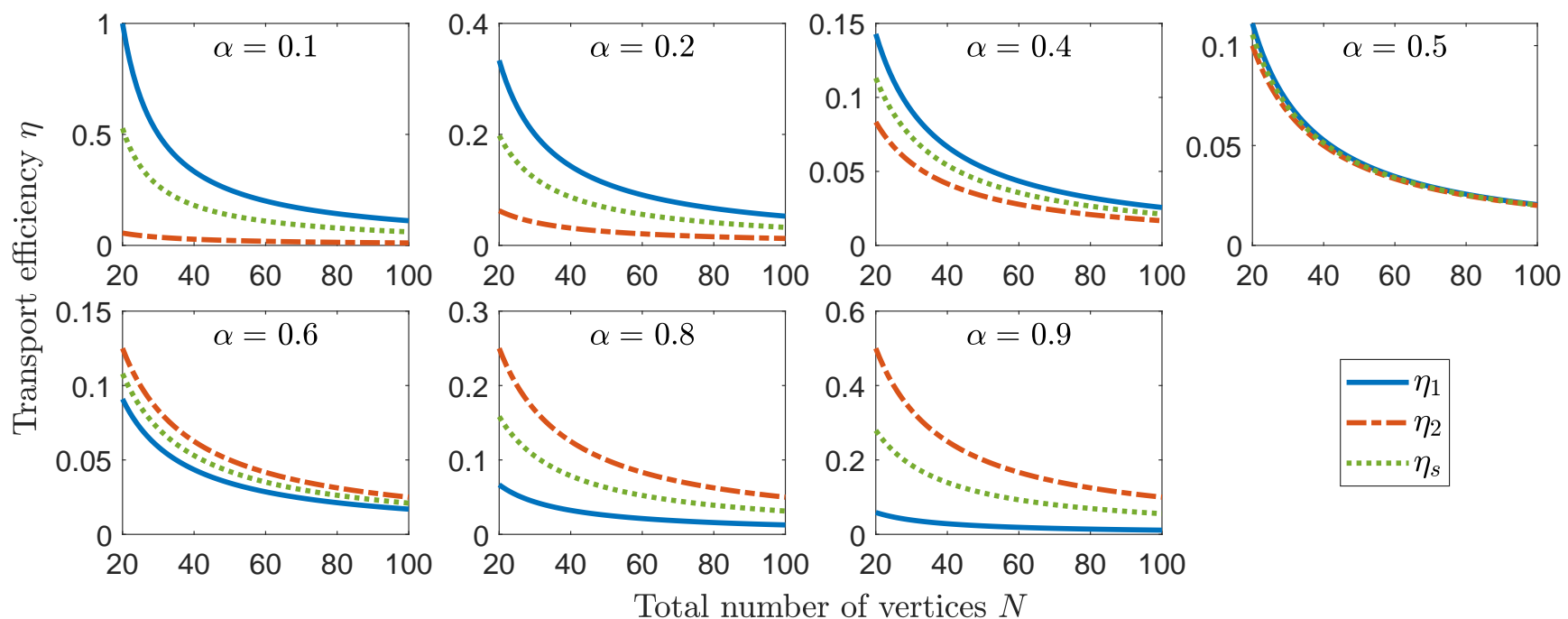

Total number of vertices $N$

Figure 3. Transport efficiency $\eta$ as a function of the order $N$ of the complete bipartite graph for different values of $\alpha=N_{1} / N$, with $N_{1}=\left|V_{1}\right|$, and different initial states. Transport efficiencies $\eta_{1(2)}$ (19) when the initial state is localized at a vertex in $V_{1(2)}$, and $\eta_{s}(21)$ when the initial state is the superposition of two vertices, one in $V_{1}$ and the other in $V_{2}$. The trap vertex $w \in V_{1}$.

\subsection{Strongly Regular Graph}

A strongly regular graph (SRG) with parameters $(N, k, \lambda, \mu)$ is a graph with $N$ vertices, not complete or edgeless, where each vertex is adjacent to $k$ vertices; for each pair of adjacent vertices, there are $\lambda$ vertices adjacent to both, and for each pair of nonadjacent vertices there are $\mu$ vertices that are adjacent to both [51,52]. If we consider the red vertex $w$ in Figure 4 , this means that there are $k$ yellow adjacent vertices, and $N-k-1$ blue vertices, all at distance 2. SRGs have a local symmetry, but most have no global symmetry [46]. The four parameters $(N, k, \lambda, \mu)$ are not independent and, for some parameters, there are no SRGs. One necessary, but not sufficient, condition is that the parameters satisfy

$$
k(k-\lambda-1)=(N-k-1) \mu,
$$

which can be proved by counting, in two wayy, the vertices at distance 0,1 , and 2 from a given vertex. Let us focus on the red vertex shown in Figure 4 and count the pairs of yellow and blue vertices that are adjacent to it. On the left-hand side of Equation (22), the red vertex has $k$ neighbors, the yellow ones. Each yellow vertex has $k$ neighbors, one of which is the red one and $\lambda$ of which are other yellow vertices, so it is adjacent to $k-\lambda-1$ blue vertices. Hence, the number of pairs of adjacent yellow and blue vertices is $k(k-\lambda-1)$. On the right-hand side of Equation (22), we consider the blue vertices, which, by definition, are not adjacent to the red vertex. There are $N-k-1$ blue vertices, since there are $N$ total vertices in the graph, one of which is red and $k$ of which are yellow. Each of the blue vertices is adjacent to $\mu$ yellow vertices, so there are $(N-k-1) \mu$ pairs of yellow and blue vertices. The condition (22) comes from equating these expressions [46].

The system evolves in a 3-dimensional subspace (see Appendix B.2) spanned by the orthonormal basis states

$$
\left|e_{1}\right\rangle=|w\rangle, \quad\left|e_{2}\right\rangle=\frac{1}{\sqrt{k}} \sum_{(i, w) \in E}|i\rangle, \quad\left|e_{3}\right\rangle=\frac{1}{\sqrt{N-k-1}} \sum_{(i, w) \notin E}|i\rangle .
$$


This is also the basis that we would obtain by grouping together the identically evolving vertices in the subsets $V_{a}=\{i \mid(i, w) \in E\}$ and $V_{b}=\{i \mid(i, w) \notin E\}$ (see Figure 4) [46]. In this subspace, the reduced Hamiltonian is

$$
H=\left(\begin{array}{ccc}
k-i \kappa & -\sqrt{k} & 0 \\
-\sqrt{k} & k-\lambda & -\sqrt{\mu(k-\lambda-1)} \\
0 & -\sqrt{\mu(k-\lambda-1)} & \mu
\end{array}\right) .
$$

If the initial state is localized at a vertex $v \neq w$, then the transport efficiency is

$$
\eta= \begin{cases}\frac{1}{k} & \text { if }(v, w) \in E, \\ \frac{1}{N-k-1} & \text { if }(v, w) \notin E .\end{cases}
$$

Instead, if the initial state is a superposition of two vertices one of which is adjacent to $w$ and the other is not, i.e., $\left(v_{1}, w\right) \in E$ and $\left(v_{2}, w\right) \notin E$, then the transport efficiency

$$
\eta_{s}=\frac{N-1}{2 k(N-k-1)}
$$

follows from Equation (16).

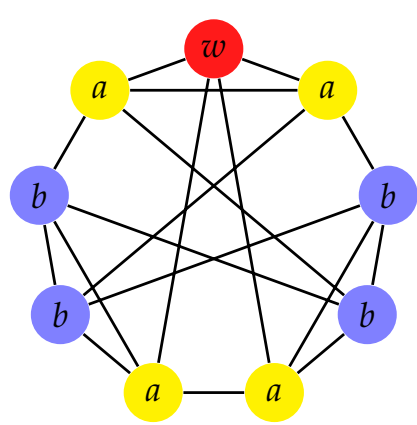

(a)

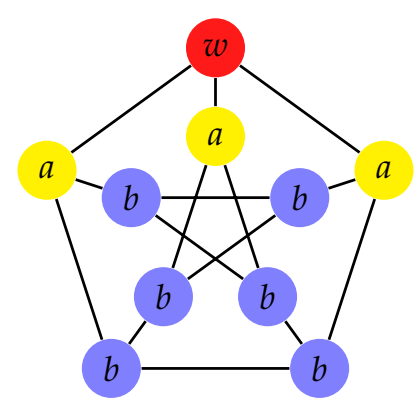

(b)

Figure 4. Two strongly regular graphs: (a) Paley graph with parameters $(9,4,1,2)$ (parametrization (27) for $\mu=2)$; (b) Petersen graph with parameters $(10,3,0,1)$. The trap vertex $w$ is colored red. Identically evolving vertices have same transport properties and are identically colored and labeled.

A family of SRGs is the Paley graphs (see Figure 4a), which are parametrized by

$$
(N, k, \lambda, \mu)=(4 \mu+1,2 \mu, \mu-1, \mu)
$$

where $N$ must be a prime power (i.e., a prime or integer power of a prime [53]) such that $N \equiv 1(\bmod 4)$. According to the parametrization (27), whether we consider an initial localized state or a superposition of two localized states, the transport efficiency on a Paley graph is $\eta=1 / 2 \mu$ (see Equations (25) and (26)), regardless of the fact that the vertices considered are adjacent or not to $w$.

\subsection{Joined Complete Graphs}

The transport efficiency on a complete graph, when the initial state is localized at a vertex $v \neq w$, is $\eta=1 /(N-1)[40,48]$. Here, we consider two complete graphs of $N / 2$ vertices that are joined by a single edge (see Figure 5 ). The two vertices, $b_{1}$ and $b_{2}$, forming the "bridge" have degree $N / 2$, whereas all of the others have degree $N / 2-1$. We denote each complete graph by $K_{N / 2}^{(k)}=\left(V_{k}, E_{k}\right)$, with $k=1,2$, where $\left|V_{1}\right|=\left|V_{2}\right|=N / 2$. Therefore, the resulting joined graph is such that $V=V_{1} \cup V_{2}$ and $E=E_{1} \cup E_{2} \cup\left\{\left(b_{1}, b_{2}\right)\right\}$. 


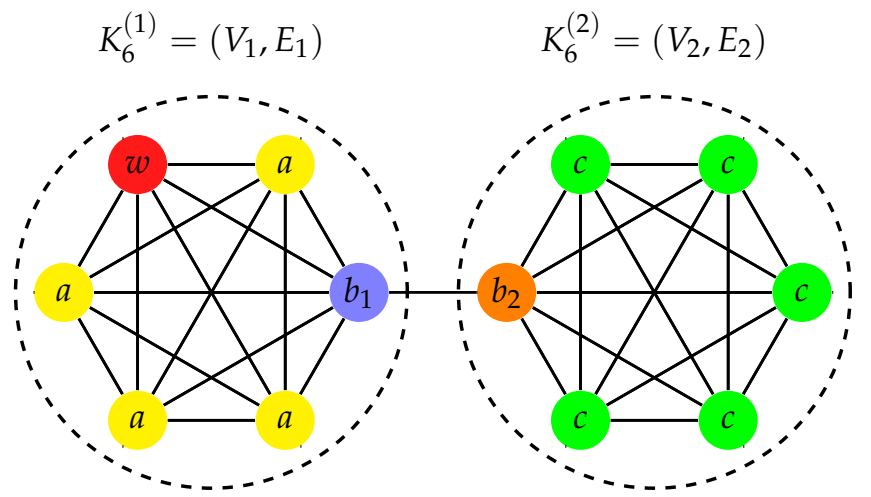

Figure 5. A graph with 12 vertices constructed by joining two complete graphs of 6 vertices by a single edge $\left(b_{1}, b_{2}\right)$, the bridge. The trap vertex $w \in V_{1}$ is colored red. Identically evolving vertices have same transport properties and are identically colored and labeled. The vertices $b_{1}$ and $b_{2}$ show the same transport efficiency, even if they behave differently under the action of the Hamiltonian.

Grouping together the identically evolving vertices, we define the subsets $V_{a}=$ $V_{1} \backslash\left\{w, b_{1}\right\}$ and $V_{c}=V_{2} \backslash\left\{b_{2}\right\}$ (see Figure 5). The system evolves in a 4-dimensional subspace (see Appendix B.3) that is spanned by the orthonormal basis states

$\left|e_{1}\right\rangle=|w\rangle$,

$\left|e_{2}\right\rangle=\frac{1}{\sqrt{N / 2-1}}\left(\sum_{i \in V_{a}}|i\rangle+\left|b_{1}\right\rangle\right)$

$\left|e_{3}\right\rangle=\frac{1}{\sqrt{(N-3)(N / 2-1)}}\left[\sum_{i \in V_{a}}|i\rangle-(N / 2-2)\left|b_{1}\right\rangle+(N / 2-1)\left|b_{2}\right\rangle\right]$,

$\left|e_{4}\right\rangle=\frac{1}{\sqrt{(N-3)[N(N / 2-2)+1]}}\left[\sum_{i \in V_{a}}|i\rangle-(N / 2-2)\left(\left|b_{1}\right\rangle+\left|b_{2}\right\rangle\right)-(N-3) \sum_{i \in V_{c}}|i\rangle\right]$.

We point out that this basis spans a subspace of dimension 4, thus smaller than the 5dimensional subspace spanned by the basis that is defined by grouping together the identically evolving vertices [47]. In the subspace that is spanned by the basis states $\left\{\left|e_{1}\right\rangle, \ldots,\left|e_{4}\right\rangle\right\}$, the reduced Hamiltonian is

$$
H=\left(\begin{array}{cccc}
N / 2-1-i \kappa & -\sqrt{N / 2-1} & 0 & 0 \\
-\sqrt{N / 2-1} & \frac{N}{N-2} & -\frac{\sqrt{N-3}}{N / 2-1} & 0 \\
0 & -\frac{\sqrt{N-3}}{N / 2-1} & \frac{1}{N-3}\left(\frac{N^{2}}{2}-7+\frac{1}{N / 2-1}\right) & \frac{\sqrt{(N / 2-1)[N(N / 2-2)+1]}}{N-3} \\
0 & 0 & \frac{\sqrt{(N / 2-1)[N(N / 2-2)+1]}}{N-3} & \frac{N / 2-1}{N-3}
\end{array}\right) .
$$

If the initial state is localized at a vertex $v \neq w$, then the transport efficiency is

$$
\eta= \begin{cases}\frac{2(N-1)}{N(N-4)+2} & \text { if } v \in V_{a}, \\ \frac{1}{2}+\frac{N-3}{N(N-4)+2} & \text { if } v \in\left\{b_{1}, b_{2}\right\}, \\ \frac{2(N-3)}{N(N-4)+2} & \text { if } v \in V_{c} .\end{cases}
$$


Assuming that each complete graph has $N / 2 \geq 3$ vertices, then $\eta_{c}<\eta_{a} \leq \eta_{b}$, where the subscript refers to an initial state localized at vertex in $V_{c}$, in $V_{a}$, and in the bridge $\left\{b_{1}, b_{2}\right\}$, respectively. Instead, if the initial state is a superposition of two vertices, then

$$
\eta_{s}(\theta)= \begin{cases}\frac{(N-2)[N+4(1+\cos \theta)]}{4[N(N-4)+2]}=\frac{1}{4}+O\left(\frac{1}{N}\right) & \text { if } v_{1} \in V_{a} \wedge v_{2} \in\left\{b_{1}, b_{2}\right\}, \\ \frac{2(N-2-\cos \theta)}{N(N-4)+2}=\frac{2}{N}+O\left(\frac{1}{N^{2}}\right) & \text { if } v_{1} \in V_{a} \wedge v_{2} \in V_{c}, \\ \frac{(N-2)[N-(N-4) \cos \theta]-4}{2[N(N-4)+2]}=\frac{1-\cos \theta}{2}+O\left(\frac{1}{N}\right) & \text { if } v_{1}=b_{1} \wedge v_{2}=b_{2}, \\ \frac{N(N+2)+4(N-4) \cos \theta-16}{4[N(N-4)+2]}=\frac{1}{4}+O\left(\frac{1}{N}\right) & \text { if } v_{1} \in\left\{b_{1}, b_{2}\right\} \wedge v_{2} \in V_{c} .\end{cases}
$$

We observe that, for the superposition of $v_{1} \in V_{a}$ and $v_{2} \in V_{c}$, the transport efficiency $\eta_{s}(\pi)$ is equal to $\eta$ for an initial state that is localized at $v \in V_{a}$. For the superposition of $b_{1}$ and $b_{2}$, i.e., of the vertices of the bridge, we have $\eta_{s}(\pi)=1$. This means that such a state belongs to $\mathcal{I}(H,|w\rangle)$, indeed

$$
\frac{1}{\sqrt{2}}\left(\left|b_{1}\right\rangle-\left|b_{2}\right\rangle\right)=\frac{1}{\sqrt{N-2}}\left(\left|e_{2}\right\rangle-\sqrt{N-3}\left|e_{3}\right\rangle\right) .
$$

For an initial state localized at $b_{1}$ or $b_{2}$, we have the same transport efficiency $\eta_{b}(30)$. However, the two vertices $b_{1}$ and $b_{2}$ have different overlap with the basis states $\left|e_{k}\right\rangle$, so the transport efficiency (31) for the superposition of them is not given by Equation (15).

\subsection{Simplex of Complete Graphs}

We call $M$-simplex of complete graphs what is formally known as the first-order truncated $M$-simplex lattice. The truncated $M$-simplex lattice is a generalization of the truncated tetrahedron lattice [54] and it is defined recursively. The graph of the zeroth order truncated $M$-simplex lattice is a complete graph of $M+1$ vertices. The graph for the $(n+1)$ th order lattice is obtained by replacing each of the vertices of the $n$th order graph with a complete graph of $M$ vertices. The truncated simplex lattice has been studied in various problems, e.g., in statistical models [55], self-avoiding random walks [56], and spatial search $[47,57]$. The $M$-simplex is, therefore, obtained by replacing each of the $M+1$ vertices of a complete graph with a complete graph of $M$ vertices (see Figure 6). Each of the new $M$ vertices is connected to one of the edges coming to the original vertex. The graph is regular, vertex transitive, and there are $N=M(M+1)$ total vertices.

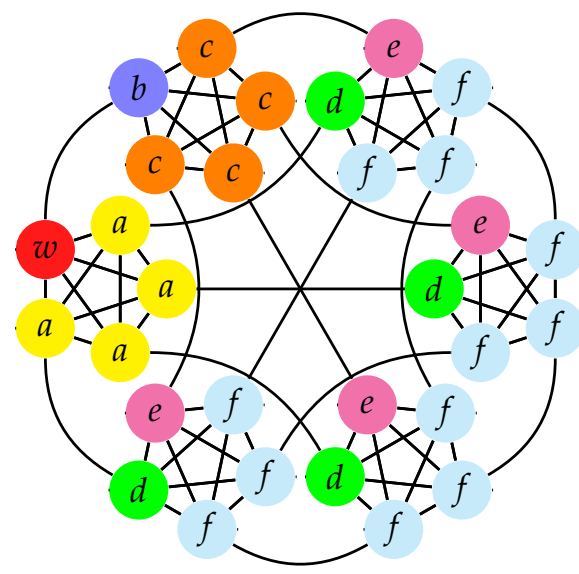

Figure 6. 5-simplex of complete graphs. The trap vertex $w$ is colored red. Identically evolving vertices have same transport properties and are identically colored and labeled. The vertices in $V_{c}$ and $V_{d}$ show the same transport efficiency, even if they behave differently under the action of the Hamiltonian. 
Grouping together the identically evolving vertices, we define the subsets $V_{a}, V_{c}$, $V_{d}, V_{e}$, and $V_{f}$ (see Figure 6), having cardinality $\left|V_{a}\right|=\left|V_{c}\right|=\left|V_{d}\right|=\left|V_{e}\right|=M-1$, and $\left|V_{f}\right|=(M-1)(M-2)$. The yellow vertices $a$ are adjacent to $w$ and belong to the same complete graph. The blue vertex $b$ is adjacent to $w$, but it belongs to a different complete graph. The orange vertices $c$ are adjacent to $b$ and belong to the same complete graph. The green vertices $d$, even if, at distance 2 from $w$, like the vertices $c$, are adjacent to $a$, and so they form a different subset. The magenta vertices $e$ are adjacent to $c$ and belong to complete graphs other than the one the vertices $c$ belong to. The cyan vertices $f$ are adjacent to $e$ and $d$. Independent of $M$, the system evolves in a 5-dimensional subspace (see Appendix B.4) that spanned by the orthonormal basis states

$$
\begin{aligned}
& \left|e_{1}\right\rangle=|w\rangle, \\
& \left|e_{2}\right\rangle=\frac{1}{\sqrt{M}}\left(\sum_{i \in V_{a}}|i\rangle+|b\rangle\right) \\
& \left|e_{3}\right\rangle=\frac{\sqrt{M}}{\sqrt{(M-1)\left(M^{2}-2 M+4\right)}}\left\{\frac{M-2}{M}\left[\sum_{i \in V_{a}}|i\rangle-(M-1)|b\rangle\right]+\sum_{i \in V_{c} \cup V_{d}}|i\rangle\right\} \text {, } \\
& \left|e_{4}\right\rangle=\frac{\sqrt{M^{2}-2 M+4}}{\sqrt{(M-1)\left(M^{3}+2 M^{2}-8 M+16\right)}}\left\{\frac{2(M-2)}{M^{2}-2 M+4}\left[\sum_{i \in V_{a}}|i\rangle-(M-1)|b\rangle\right]\right. \\
& \left.-\frac{(M-2)^{2}}{M^{2}-2 M+4} \sum_{i \in V_{c} \cup V_{d}}|i\rangle-2 \sum_{i \in V_{e}}|i\rangle-\sum_{i \in V_{f}}|i\rangle\right\} \text {, } \\
& \left|e_{5}\right\rangle=\frac{1}{M \sqrt{(M-1)(M-2)\left(M^{3}+2 M^{2}-8 M+16\right)}}\left\{-4(M-2)\left[\sum_{i \in V_{a}}|i\rangle-(M-1)|b\rangle\right]\right. \\
& \left.+2(M-2)^{2} \sum_{i \in V_{c} \cup V_{d}}|i\rangle-M^{2}(M-2) \sum_{i \in V_{e}}|i\rangle+2\left(M^{2}-2 M+4\right) \sum_{i \in V_{f}}|i\rangle\right\} \text {. }
\end{aligned}
$$

Note that, when the basis states include the vertices in $V_{c}$ and $V_{d}$, they always involve the equal superposition of all the vertices in $V_{c} \cup V_{d}$. Thus, these vertices are equivalent for quantum transport, even if they behave differently under the action of the Hamiltonian. We point out that this basis spans a subspace of dimension 5, thus being smaller than the 7-dimensional subspace spanned by the basis that is defined by grouping together the identically evolving vertices $[47,50]$. In the subspace that is spanned by the basis states $\left\{\left|e_{1}\right\rangle, \ldots,\left|e_{5}\right\rangle\right\}$, the reduced Hamiltonian is a symmetric tridiagonal matrix with cumbersome elements, so we store the main diagonal and the superdiagonal, as follows

$$
\left(\begin{array}{cc}
H_{1,1} & H_{1,2} \\
\vdots & \vdots \\
H_{n, n} & H_{n, n+1} \\
\vdots & \vdots \\
H_{5,5} & *
\end{array}\right)=\left(\begin{array}{cc}
M-i \kappa & -\sqrt{M} \\
\frac{3 M-2}{M} & -\frac{\sqrt{(M-1)\left(M^{2}-2 M+4\right)}}{M} \\
\frac{M^{4}-2 M^{3}+4 M^{2}-4 M+8}{M\left(M^{2}-2 M+4\right)} & \frac{\sqrt{M\left(M^{3}+2 M^{2}-8 M+16\right)}}{M^{2}-2 M+4} \\
\frac{M\left(M^{4}-2 M^{3}+20 M^{2}-40 M+64\right)}{\left(M^{3}+2 M^{2}-8 M+16\right)\left(M^{2}-2 M+4\right)} & \frac{M(M+2) \sqrt{(M-2)\left(M^{2}-2 M+4\right)}}{M^{3}+2 M^{2}-8 M+16} \\
\frac{(M+2)\left(M^{3}-4 M+8\right)}{M^{3}+2 M^{2}-8 M+16} & *
\end{array}\right),
$$

where the $*$ denotes the missing element, because its index exceeds the size of the matrix. 
If the initial state is localized at a vertex $v \neq w$, then the transport efficiency is

$$
\eta= \begin{cases}\frac{M^{2}-2}{M^{2}(M-1)} & \text { if } v \in V_{a}, \\ \frac{M^{2}-2 M+2}{M^{2}} & \text { if } v=b, \\ \frac{2}{M^{2}} & \text { if } v \in V_{c} \cup V_{d}, \\ \frac{1}{M-1} & \text { if } v \in V_{e}, \\ \frac{M^{2}-2 M+4}{M^{2}(M-1)(M-2)} & \text { if } v \in V_{f} .\end{cases}
$$

Note that, for an initial state localized at $b$, which is the only vertex adjacent to $w$ which does not belong to the complete graph of $w$ (see Figure 6), we have $\eta_{b} \approx 1$ for large $M$. Instead, if the initial state is a superposition of two vertices, then

$$
\eta_{s}(\theta)= \begin{cases}\frac{M\left(M^{2}-2 M+4\right)-4+4(M-1) \cos \theta}{2 M^{2}(M-1)}=\frac{1}{2}+O\left(\frac{1}{M}\right) & \text { if } v_{1} \in V_{a} \wedge v_{2}=b, \\ \frac{M^{2}+2 M-4+2(M-2) \cos \theta}{2 M^{2}(M-1)}=\frac{1}{2 M}+O\left(\frac{1}{M^{2}}\right) & \text { if } v_{1} \in V_{a} \wedge v_{2} \in V_{c} \cup V_{d}, \\ \frac{1}{M}+\frac{1}{M^{2}} & \text { if } v_{1} \in V_{a} \wedge v_{2} \in V_{e}, \\ \frac{M\left(M^{2}-M-4\right)+8-4(M-2) \cos \theta}{2 M^{2}(M-1)(M-2)}=\frac{1}{2 M}+O\left(\frac{1}{M^{2}}\right) & \text { if } v_{1} \in V_{a} \wedge v_{2} \in V_{f}, \\ \frac{1}{M^{2}-\frac{1}{M}+\frac{M}{2(M-1)}=\frac{1}{2}+O\left(\frac{1}{M}\right)} & \text { if } v_{1}=b \wedge v_{2} \in V_{c} \cup V_{d}, \\ \frac{M\left(M^{3}-5 M^{2}+11 M-12\right)+8}{2 M^{2}(M-1)(M-2)}+\frac{2}{M^{2}} \cos \theta=\frac{1}{2}+O\left(\frac{1}{M}\right) & \text { if } v_{1}=b \wedge v_{2} \in V_{e}, \\ \frac{1}{M^{2}}+\frac{1}{2(M-1)}=\frac{1}{2 M}+O\left(\frac{1}{M^{2}}\right) & \text { if } v_{1} \in v_{c} \cup v_{d} \wedge V_{f}, \\ \frac{3 M^{2}-8 M+8+2(M-2)^{2} \cos \theta}{2 M^{2}(M-1)(M-2)}=\frac{3 / 2+\cos \theta}{M^{2}}+O\left(\frac{1}{M^{3}}\right) & \text { if } v_{1} \in V_{c} \cup V_{d} \wedge v_{2} \in V_{f}, \\ \frac{1}{M^{2}}+\frac{1}{M}-\frac{1}{M-1}+\frac{1}{2(M-2)}=\frac{1}{2 M}+O\left(\frac{1}{M^{2}}\right) & \text { if } v_{1} \in V_{e} \wedge v_{2} \in V_{f} .\end{cases}
$$

Whenever the superposition of two vertices involves the vertex $b$, we have $\eta_{s} \approx 1 / 2$ for large $M$ and, in particular, $\eta_{s}(\pi)=1 / 2$ for $v_{1}=b \wedge v_{2} \in V_{c} \cup V_{d}$, independent of $M$ (see Figure 7). Whenever the superposition involves a vertex in $V_{e}$, the transport efficiency does not depend on $\theta$. Moreover, we observe that the equal superposition of the vertices in $V_{e}$ belongs to $\mathcal{I}(H,|w\rangle$, since

$$
\frac{1}{\sqrt{M-1}} \sum_{i \in V_{e}}|i\rangle=-\frac{1}{\sqrt{M^{3}+2 M^{2}-8 M+16}}\left(2 \sqrt{M^{2}-2 M+4}\left|e_{4}\right\rangle+M \sqrt{M-2}\left|e_{5}\right\rangle\right),
$$

and so this state provides $\eta=1$.

In the $M$-simplex of complete graphs, the total number vertices is $N=M(M+1)$, so the asymptotic behavior of the transport efficiency must be understood, according to $M=O(\sqrt{N})$. 


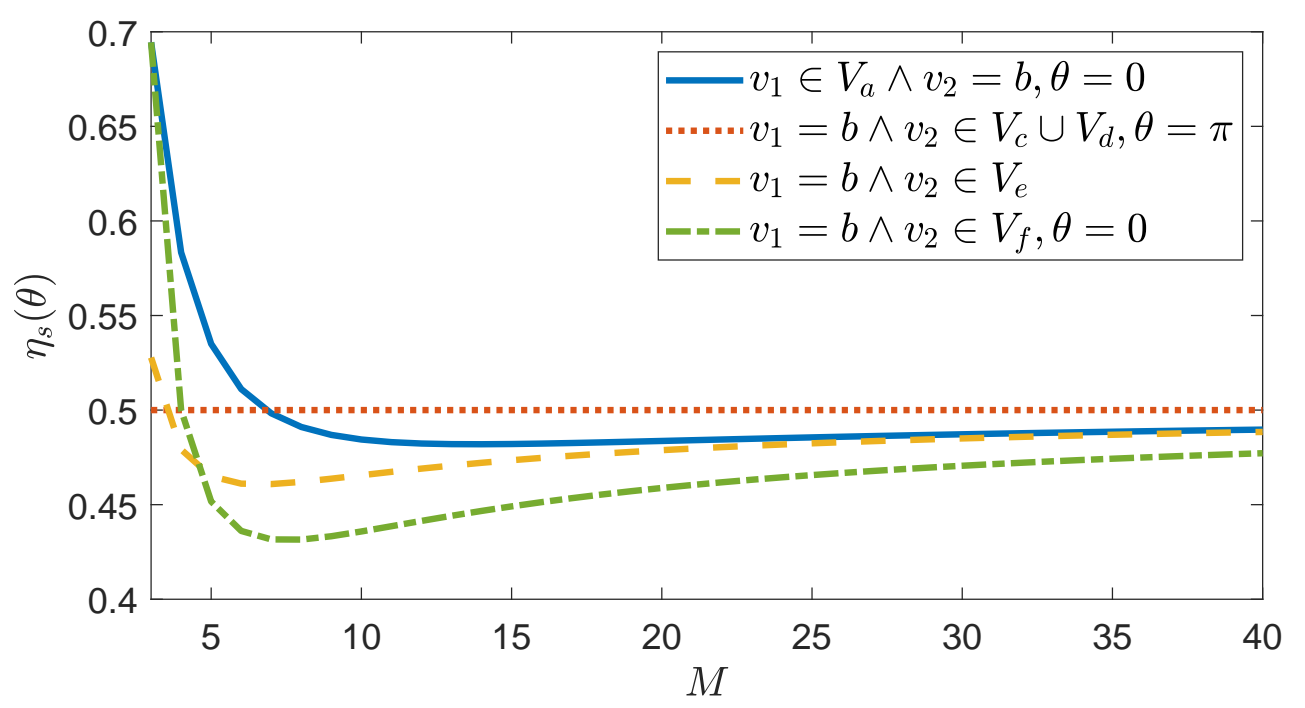

Figure 7. Transport efficiency $\eta_{s}(\theta)$ (36) as a function of $M$ for different initial states $\left|\psi_{0}\right\rangle=\left(\left|v_{1}\right\rangle+\right.$ $\left.e^{i \theta}\left|v_{2}\right\rangle\right) / \sqrt{2}$. $M$ is the number of vertices in each of the $M+1$ complete graphs forming the $M$ simplex. The initial states are the possible equal superposition of two vertices, one of which is $b$.

\section{Measures of Connectivity}

The vertex connectivity $v(G)$ and edge connectivity $e(g)$ of a graph $G$ are, respectively, the number of vertices or edges that we must remove to make $G$ disconnected [58]. These are the two most common measures of graph connectivity, and

$$
v(G) \leq e(G) \leq \delta(G)
$$

i.e., both $v(G)$ and $e(G)$ are upper bounded by the minimum degree of the graph $\delta(G)$ [59].

Another measure follows from the Laplace spectrum of the graph. The secondsmallest eigenvalue $a(G)$ of the Laplacian of a graph $G$ with $N \geq 2$ vertices is the algebraic connectivity $[60,61]$ and, to a certain extent, it is a good parameter to measure how well a graph is connected. In spectral graph theory it is well known, e.g., that a graph is connected if and only if its algebraic connectivity is different from zero. Indeed, the multiplicity of the Laplace eigenvalue zero of an undirected graph $G$ is equal to the number of connected components of $G$ [52]. For a complete graph, we know that $v\left(K_{N}\right)=e\left(K_{N}\right)=N-1$ and $a\left(K_{N}\right)=N$. Instead, for a noncomplete graph $G$, we have $a(G) \leq v(G)$, and so $a(G) \leq e(G)[58]$.

The results of the different measures of connectivity for each graph are shown in Table 1. Vertex, edge, and algebraic connectivities for the complete and the complete bipartite graphs are from [58]. The measures of connectivity for the $M$-simplex of complete graphs are from [47].

The vertex connectivity of a SRG is $v(G)=k$ [52] and the edge connectivity is $e(G)=k$. The latter follows from Equation (38), since $\delta(G)=k$, or using the fact that, if a graph has diameter 2, as the SRG has [62], then $e(G)=\delta(G)$ [59]. We need the Laplace spectrum in order to assess the algebraic connectivity. The eigenvalues of the adjacency matrix $A$ are

$$
\frac{1}{2}\left[\lambda-\mu \pm \sqrt{(\lambda-\mu)^{2}+4(k-\mu)}\right], \quad k,
$$

and the scaling of them with $N$ depends on the type of SRG. Indeed, SRGs can be classified into two types $[51,59,62]$. Type I graphs, for which $(N-1)(\mu-\lambda)=2 k$. This implies that $\lambda=\mu-1, k=2 \mu$, and $N=4 \mu+1$. They exist if and only if $N$ is the sum of two squares. Examples include the Paley graphs (see parametrization (27)). Type II graphs, for which $(\mu-\lambda)^{2}+4(k-\mu)$ is a perfect square $d^{2}$, where $d$ divides $(N-1)(\mu-\lambda)-2 k$, and the quotient is congruent to $N-1(\bmod 2)$. Type I graphs are also type II graphs if and 
only if $N$ is a square [51]. The Paley graph $(9,4,1,2)$ is an example of this (see Figure $4 a$ ). Not all of the SRGs of type II are known, only certain parameter families, e.g., the Latin square graphs [51], and certain graphs, e.g., the Petersen graph (see Figure 4b), are. Hence, we consider the algebraic connectivity only for the SRGs of type I. According to the parametrization of the SRG of type I and to the fact that $D=k I$, the eigenvalues of $L=D-A$ are

$$
0, \quad \frac{1}{2}(N \mp \sqrt{N}),
$$

from which the algebraic connectivity is $a(G)=(N-\sqrt{N}) / 2$, since $\mu=(N-1) / 4$ and $k=(N-1) / 2$.

Table 1. The minimum degrees and vertex, edge, and algebraic connectivities of the graphs with $N$ vertices that are considered in this work. For these graphs, the vertex and the edge connectivities are equal. Note that, in the $M$-simplex of complete graphs, $N=M(M+1)$.

\begin{tabular}{cccc}
\hline Graph $G$ & $\delta(G)$ & $v(G)=e(G)$ & $a(G)$ \\
\hline Complete $K_{N}$ & $N-1$ & $N-1$ & $N$ \\
Complete bipartite $K_{N_{1}, N_{2}}$ & $\min \left(N_{1}, N_{2}\right)$ & $\min \left(N_{1}, N_{2}\right)$ & $\min \left(N_{1}, N_{2}\right)$ \\
Strongly regular (Type I) & $(N-1) / 2$ & $(N-1) / 2$ & $(N-\sqrt{N}) / 2$ \\
Joined complete $K_{N / 2}$ & $N / 2-1$ & 1 & $O(1 / N)$ \\
$M$-simplex & $M=O(\sqrt{N})$ & $M=O(\sqrt{N})$ & 1 \\
\hline
\end{tabular}

For the joined complete graphs we have $v(G)=e(G)=1$, because of the bridge (see Figure 5) [63]. The Laplace spectrum is

$$
0, \frac{N}{2}, \frac{1}{4}[N+4 \pm \sqrt{N(N+8)-16}]
$$

from which the algebraic connectivity is $a(G)=[N+4-\sqrt{N(N+8)-16}] / 4$.

Subsequently, we assess whether connectivity of the graph may provide or not some bounds on the transport efficiency for an initial state localized at a vertex. First, we focus on the regular graphs considered in this work, for which $\delta(G)=v(G)=e(G)$, and this is equal to the degree. For a complete graph, we have $1 / a(G) \leq \eta=1 /(N-1)$, and $1 /(N-1)$ is also the reciprocal of the degree. For a SRG of type I, we have $\eta=2 /(N-1) \leq 1 / a(G)$ for $\mu \geq 1$, and $2 /(N-1)$ is also the reciprocal of the degree. Hence, from these two examples, we see that the reciprocal of the algebraic connectivity does not provide a common bound on $\eta$. For the $M$-simplex of complete graphs, we observe that $a(G)=1$, from whose reciprocal we obtain the obvious upper bound $\eta \leq 1$. Note also that, in general, the transport efficiency for an initial state that is localized at vertex of a regular graph is not the reciprocal of the degree, as shown, e.g., by the transport efficiency on a general SRG (25) (degree $k$ ) and on the $M$-simplex (35) (degree $M$ ).

Now, we focus on the non-regular graphs. For the joined complete graphs, the reciprocal of the vertex and edge connectivity provides the obvious bound $\eta \leq 1$, whereas neither the reciprocal of $\delta(G)$ nor that of $a(G)$ provide a unique bound on $\eta$. Indeed, they are an upper or lower bound on $\eta$, depending on the initial state and the order of the graph (see Equation (30)). For the CBG, the vertex, edge, and algebraic connectivity is $\min \left(N_{1}, N_{2}\right)$ and its reciprocal is an upper or lower bound on the transport efficiency (19), depending on the geometry of the graph. Indeed, we have $\eta_{1} \leq \eta_{2} \leq 1 / \min \left(N_{1}, N_{2}\right)$ for $\alpha>1 / 2$, i.e., $N_{1}>N_{2}$, and $1 / \min \left(N_{1}, N_{2}\right)=\eta_{2} \leq \eta_{1}$ for $\alpha \leq 1 / 2$, i.e., $N_{1} \leq N_{2}$.

In conclusion, just by focusing on the transport efficiency for an initial state localized at a vertex, we observe that the connectivity is a poor indicator for the transport efficiency. First, because it does not provide any general lower or upper bound for estimating the transport efficiency, and transport efficiency and connectivity are generally uncorrelated (see Figure 8). Second, because transport efficiency strongly depends on the initial state, or, rather, on the overlap of this with the subspace spanned by the eigenstates of the 
Hamiltonian having non-zero overlap with the trap vertex, as shown in Section 4. Note that, analogously, we have found no general correlation between the transport efficiency and normalized algebraic connectivity, which is the second-smallest eigenvalue of the normalized Laplacian matrix $\mathcal{L}$ of elements $\mathcal{L}_{j k}=L_{j k} / \sqrt{\operatorname{deg}(j) \operatorname{deg}(k)}$ [64].
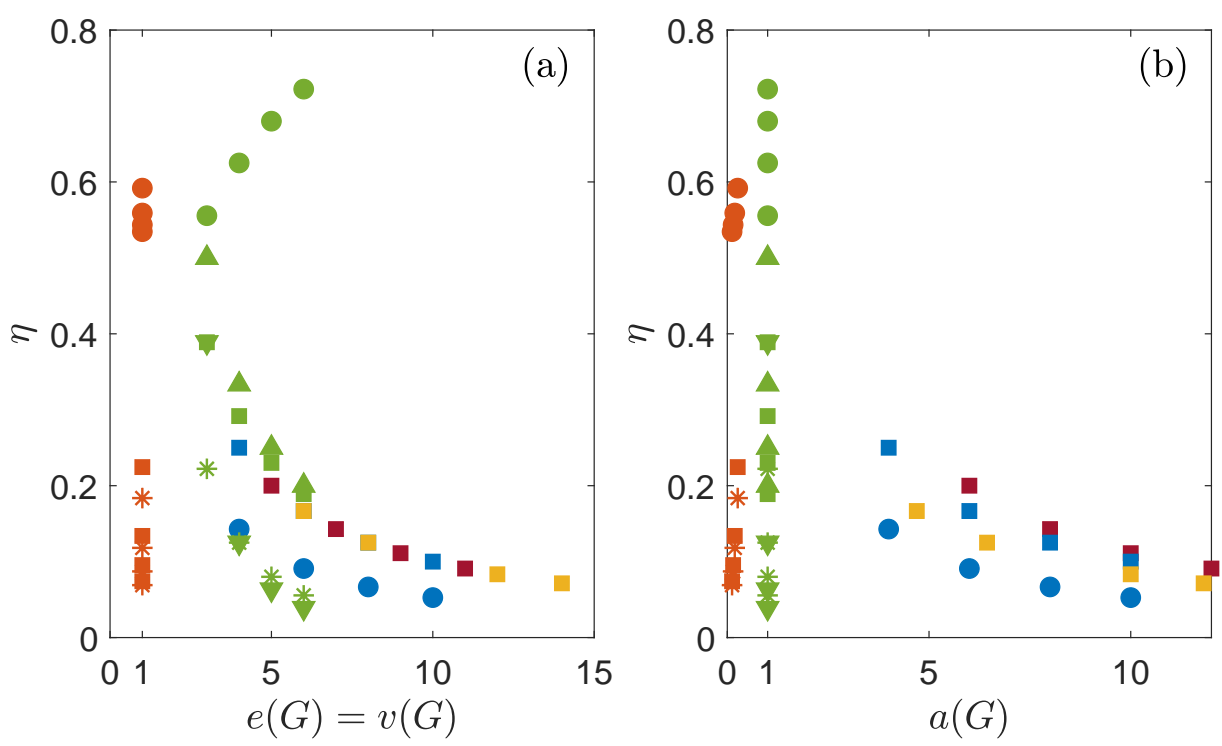

\begin{tabular}{|c|}
\hline $\begin{array}{l}\text { CG } \\
\text { CBG } v \in V_{2} \\
\text { CBG } v \in V_{1} \backslash\{w\} \\
\text { SRG (Type I) } \\
\text { JCG } v \in V_{a} \\
\text { JCG } v \in\left\{b_{1}, b_{2}\right\} \\
\text { JCG } v \in V_{c} \\
\text { SCG } v \in V_{a} \\
\text { SCG } v=b \\
\text { SCG } v \in V_{c} \cup V_{d} \\
\text { SCG } v \in V_{e} \\
\text { SCG } v \in V_{f}\end{array}$ \\
\hline
\end{tabular}

Figure 8. Scatter plot of the correlation between the transport efficiency $\eta$ and (a) the edge or vertex connectivity, $e(G)$ and $v(G)$ respectively, or $(\mathbf{b})$ the algebraic connectivity $a(G)$ (see also Table 1). Same color denotes results for the same graph: complete graph (CG, $N=6,8,10,12)$, complete bipartite graph (CBG, $N=12,18,24,30, \alpha=2 / 3$ ), strongly regular graphs of type I (SRG, $N=13,17,25,29)$, joined complete graphs (JCG, $N=12,18,24,30)$, and $M$-simplex of complete graphs (SCG, $M=3,4,5,6)$. For a given a graph, different markers denote initial states localized at different vertices $v$. Note that, for the SRG of type I $\eta=1 / 2 \mu=2 /(N-1)$, independent of the fact that $(v, w) \in E$ or $(v, w) \notin E$. We observe some specific correlations between the transport efficiency and connectivity for a given graph, but, globally, among different graphs, transport efficiency and connectivity are uncorrelated.

\section{Conclusions}

In this work, we have addressed the coherent dynamics of transport processes on graphs in the framework of continuous-time quantum walks. We have considered graphs having different properties in terms of regularity, symmetry, and connectivity, and we have modeled the loss processes via the absorbing of the wavefunction component at a single trap vertex $w$. We have adopted the transport efficiency as a figure of merit in order to assess the transport properties of the system. In the ideal regime, as the one we have adopted, where there is no disorder or decoherence processes during the transport, the transport efficiency $\eta$ can be computed as the overlap of the initial state with the subspace $\Lambda(H,|w\rangle)$ spanned by the eigenstates of the Hamiltonian having non-zero overlap with the trap vertex. According to the dimensionality reduction method, we have determined the orthonormal basis of such subspace with no need to diagonalize the Hamiltonian. Therefore, any initial state that is a linear combination of such basis states provides the maximum transport efficiency $\eta=1$. We have considered, as the initial state, either a state localized at a vertex or a superposition of two vertices, and computed the corresponding transport efficiency. Overall, the most promising graph seems to be the $M$-simplex of complete graphs, since it allows for us to have a transport efficiency that is close to 1 for large $M$ for an initially localized state. Transport with maximum efficiency is also possible on other graphs, if the walker is initially prepared in a suitable superposition state. However, the coherence of these preparations is likely to be degraded by noise, and the corresponding transport efficiency may be hard to be achieved in practice. 
Our results suggest that connectivity of the graph is a poor indicator for the transport efficiency. Indeed, we observe some specific correlations between transport efficiency and connectivity for certain graphs, but in general they are uncorrelated. Moreover, transport efficiency depends on the overlap of the initial state with $\Lambda(H,|w\rangle)$, and the reciprocal of the measures of connectivity that we have assessed does not provide a general and consistent either lower or upper bound on $\eta$. However, the topology of the graph is encoded in the Laplacian matrix, which contributes to defining the Hamiltonian. Thus, connectivity somehow affects the transport properties of the system in the sense that it affects the Hamiltonian.

On the other hand, the transport efficiency is the integrated probability of trapping in the limit of infinite time, thus other figures of merit for the transport properties, such as the transfer time, which is the average time that is required by the walker to get absorbed at the trap, and the survival probability might highlight the role of the connectivity of the graph, if any. Moreover, the role of the trap needs to be further investigated, when considering more than one trap vertex, different trapping rates, and different trap location. Our analytical results are proposed as a reference for further studies on the transport properties of these systems and as a benchmark for studying environment-assisted quantum transport on such graphs. Indeed, our work paves the way for further investigation, including the analysis of more realistic systems in the presence of noise.

Author Contributions: Conceptualization, L.R., M.G.A.P. and P.B.; Methodology, L.R., M.G.A.P. and P.B.; Software, L.R.; Validation, L.R., M.G.A.P. and P.B.; Formal analysis, L.R., M.G.A.P. and P.B.; Investigation, L.R.; Writing—original draft preparation, L.R.; Writing-review and editing, L.R., M.G.A.P. and P.B.; Visualization, L.R.; Supervision, M.G.A.P. and P.B. All authors have read and agreed to the published version of the manuscript.

Funding: This research received no external funding.

Acknowledgments: Paolo Bordone and Matteo G. A. Paris are members of GNFM-INdAM.

Conflicts of Interest: The authors declare no conflict of interest.

\section{Abbreviations}

The following abbreviations are used in this manuscript:

CTQW Continuous-time quantum walk

CBG Complete bipartite graph

SRG Strongly regular graph

Appendix A. Subspace of the Eigenstates of the Hamiltonian with Non-Zero Overlap with the Trap

In this appendix, we show that the subspace $\Lambda(H,|w\rangle)$ of the eigenstates of the Hamiltonian having nonzero overlap with the trap is equal to the subspace $\mathcal{I}(H,|w\rangle)=$ $\operatorname{span}\left(\left\{H^{k}|w\rangle \mid k \in \mathbb{N}_{0}\right\}\right)$ introduced in Section 3. This proof is from the Supplementary information of [48]. We report it for sake of completeness and because we refine a key point, not addressed in the original proof, about the right and the left inverse of a matrix.

Let $\Lambda(H,|w\rangle)=\operatorname{span}\left(\left\{\left|\lambda_{1}\right\rangle, \ldots,\left|\lambda_{m}\right\rangle\right\}\right)$, where $H\left|\lambda_{k}\right\rangle=\lambda_{k}\left|\lambda_{k}\right\rangle$ and $m$ is the minimum number of eigenstates of $H$ having non-zero overlap with the trap, i.e., $\left\langle w \mid \lambda_{k}\right\rangle \neq 0$. In case of a degenerate eigenspace, more than one eigenstate belonging to it can have a non-zero overlap with $|w\rangle$, hence the need to find the minimum number $m$. The ambiguity is solved as follows. We choose the eigenstate from this degenerate eigenspace having the maximum overlap with $|w\rangle$, then we orthogonalize all the remaining eigenstates within such eigenspace with respect to it. After orthogonalizing, these eigenstates have zero overlap with $|w\rangle[40,48]$. 
Let $\operatorname{dim}(\mathcal{I}(H,|w\rangle))=m_{1}, \operatorname{dim}(\Lambda(H,|w\rangle))=m_{2}$, and $N$ the dimension of the complete Hilbert space. First, we prove that $\mathcal{I}(H,|w\rangle) \subseteq \Lambda(H,|w\rangle)$, i.e., that any state $H^{i}|w\rangle \in \mathcal{I}(H,|w\rangle)$ also belongs to $\Lambda(H,|w\rangle)$ :

$$
H^{i}|w\rangle=\sum_{k=1}^{N}\left\langle\lambda_{k} \mid w\right\rangle H^{i}\left|\lambda_{k}\right\rangle=\sum_{k=1}^{m_{2}}\left\langle\lambda_{k} \mid w\right\rangle H^{i}\left|\lambda_{k}\right\rangle=\sum_{k=1}^{m_{2}}\left\langle\lambda_{k} \mid w\right\rangle \lambda_{k}^{i}\left|\lambda_{k}\right\rangle,
$$

since $\left\langle\lambda_{k} \mid w\right\rangle=0$ for $m_{2}+1 \leq k \leq N$. Any state $H^{i}|w\rangle$ can therefore be expressed as a linear combination of the eigenstates of the Hamiltonian having a non-zero overlap with the trap, so $H^{i}|w\rangle \in \Lambda(H,|w\rangle) \forall i \in \mathbb{N}_{0}$. Second, we prove that $\Lambda(H,|w\rangle) \subseteq \mathcal{I}(H,|w\rangle)$, i.e., that any state of $\Lambda(H,|w\rangle)$ can be expressed as a linear combination of the states of $\mathcal{I}(H,|w\rangle)$. We can write

$$
\left|\lambda_{j}\right\rangle=\sum_{i=1}^{m_{1}} c_{j i} H^{i-1}|w\rangle=\sum_{k=1}^{m_{2}} \sum_{i=1}^{m_{1}} c_{j i} \lambda_{k}^{i-1}\left\langle\lambda_{k} \mid w\right\rangle\left|\lambda_{k}\right\rangle=\sum_{k=1}^{m_{2}} \sum_{i=1}^{m_{1}} c_{j i} M_{i k}\left|\lambda_{k}\right\rangle,
$$

with matrix element $M_{i k}=\lambda_{k}^{i-1}\left\langle\lambda_{k} \mid w\right\rangle$, provided that $\sum_{i=1}^{m_{1}} c_{j i} M_{i k}=\delta_{j k}$. In terms of matrices, this condition is $C_{m_{2} \times m_{1}} M_{m_{1} \times m_{2}}=I_{m_{2} \times m_{2}}$, which means that $C$ is the left inverse of $M$, i.e., $C=M_{L}^{-1}$. Analogously, rewriting Equation (A1) and then using the first equality of Equation (A2), we have

$$
H^{j-1}|w\rangle=\sum_{i=1}^{m_{2}}\left\langle\lambda_{i} \mid w\right\rangle \lambda_{i}^{j-1}\left|\lambda_{i}\right\rangle=\sum_{i=1}^{m_{2}} M_{j i}\left|\lambda_{i}\right\rangle=\sum_{i=1}^{m_{2}} \sum_{k=1}^{m_{1}} M_{j i} c_{i k} H^{k-1}|w\rangle,
$$

provided that $\sum_{i=1}^{m_{2}} M_{j i} c_{i k}=\delta_{j k}$. In terms of matrices, this condition is $M_{m_{1} \times m_{2}} C_{m_{2} \times m_{1}}=$ $I_{m_{1} \times m_{1}}$, which means that $C$ is the right inverse of $M$, i.e., $C=M_{R}^{-1}$. Therefore, $M$ has a left and a right inverse, so $M$ must be square, $m_{1}=m_{2}=m$, and $M_{L}^{-1}=M_{R}^{-1}=M^{-1}=C$ is unique [65]. The condition under which $\Lambda(H,|w\rangle) \subseteq \mathcal{I}(H,|w\rangle)$ is thus that $M$ must be a $m \times m$ invertible matrix. The matrix $M$ is invertible if $\operatorname{det}(M) \neq 0$. We define two $m \times m$ matrices, $V_{i j}=\lambda_{j}^{i-1}$ and the diagonal matrix $D_{i j}=\delta_{i j}\left\langle\lambda_{j} \mid w\right\rangle$, such that $M=V D$. Since $\left\langle\lambda_{j} \mid w\right\rangle=0$ for $1 \leq j \leq m$, then $\operatorname{det}(V) \neq 0$. The matrix $V$ is of the Vandermonde form, so $\operatorname{det}(V)=\prod_{1 \leq i<j \leq m}\left(\lambda_{i}-\lambda_{j}\right)$. This determinant is non-zero, since all of the states $\left|\lambda_{k}\right\rangle$, for $1 \leq k \leq m$, belong to different eigenspaces, so all the $\lambda_{k}$ are different from each other. Hence, $\operatorname{det}(M)=\operatorname{det}(V) \operatorname{det}(D) \neq 0$, so $M$ is always invertible and this condition ensures that $\Lambda(H,|w\rangle) \subseteq \mathcal{I}(H,|w\rangle)$. This concludes the proof that $\Lambda(H,|w\rangle)=\mathcal{I}(H,|w\rangle)$.

\section{Appendix B. Basis of $\mathcal{I}(H,|w\rangle)$ for Each Graph}

In this appendixm we analytically derive the orthonormal basis $\left\{\left|e_{k}\right\rangle\right\}$ spanning the subspace $\mathcal{I}(H,|w\rangle)$ for each graph considered. The first basis element is $\left|e_{1}\right\rangle=|w\rangle$, the trap vertex, and the $k$-th element $\left|e_{k}\right\rangle$ is obtained by orthonormalizing (O.N.) $H\left|e_{k-1}\right\rangle$ with respect to the subspace spanned by $\left\{\left|e_{1}\right\rangle, \ldots,\left|e_{k-1}\right\rangle\right\}$. The procedure stops when we find the minimum $m$, such that $H\left|e_{m}\right\rangle \in \operatorname{span}\left(\left\{\left|e_{1}\right\rangle, \ldots,\left|e_{m}\right\rangle\right\}\right)$. The Hamiltonian (10) is the sum of the Laplacian matrix, generating the CTQW on the graph, and the trapping Hamiltonian (9), which projects onto the trap $|w\rangle$ with proper coefficient.

Appendix B.1. Complete Bipartite Graph

The Laplacian matrix of the CBG $K_{N_{1}, N_{2}}$ is

$$
L=N_{2} \sum_{i \in V_{1}}|i\rangle\left\langle i\left|+N_{1} \sum_{j \in V_{2}}\right| j\right\rangle\langle j|-\sum_{i \in V_{1}} \sum_{j \in V_{2}}(|i\rangle\langle j|+| j\rangle\langle i|),
$$


since $\operatorname{deg}\left(i \in V_{1}\right)=N_{2}$ and $\operatorname{deg}\left(j \in V_{2}\right)=N_{1}$ (see Figure 2). The basis states (17) are obtained, as follows:

$$
\begin{aligned}
H\left|e_{1}\right\rangle & =\left(N_{2}-i \kappa\right)|w\rangle-\sum_{j \in V_{2}}|j\rangle=\left(N_{2}-i \kappa\right)\left|e_{1}\right\rangle-\sqrt{N_{2}}\left|e_{2}\right\rangle \stackrel{\text { O.N. }}{\longrightarrow}\left|e_{2}\right\rangle, \\
H\left|e_{2}\right\rangle & =\frac{N_{1}}{\sqrt{N_{2}}} \sum_{j \in V_{2}}|j\rangle-\frac{1}{\sqrt{N_{2}}} \sum_{i \in V_{1}} \sum_{j \in V_{2}}|i\rangle=N_{1}\left|e_{2}\right\rangle-\sqrt{N_{2}} \sum_{\substack{i \in V_{1}, i \neq w}}|i\rangle-\sqrt{N_{2}}\left|e_{1}\right\rangle \\
& =N_{1}\left|e_{2}\right\rangle-\sqrt{N_{2}\left(N_{1}-1\right)}\left|e_{3}\right\rangle-\sqrt{N_{2}}\left|e_{1}\right\rangle \stackrel{\text { O.N. }}{\longrightarrow}\left|e_{3}\right\rangle, \\
H\left|e_{3}\right\rangle & =\frac{N_{2}}{\sqrt{N_{1}-1}} \sum_{\substack{i \in V_{1} \\
i \neq w}}|i\rangle-\frac{1}{\sqrt{N_{1}-1}} \sum_{\substack{i \in V_{1} \\
i \neq w}} \sum_{j \in V_{2}}|j\rangle=N_{2}\left|e_{3}\right\rangle-\sqrt{N_{2}\left(N_{1}-1\right)}\left|e_{2}\right\rangle .
\end{aligned}
$$

In conclusion, any state $H^{k}|w\rangle \in \operatorname{span}\left(\left\{\left|e_{1}\right\rangle,\left|e_{2}\right\rangle,\left|e_{3}\right\rangle\right\}\right) \forall k \in \mathbb{N}_{0}$, thus the states (17) form an orthonormal basis for the subspace $\mathcal{I}(H,|w\rangle)$.

Appendix B.2. Strongly Regular Graph

The Laplacian matrix of the SRG with parameters $(N, k, \lambda, \mu)$ is

$$
L=k I-\sum_{(j, i) \in E}|j\rangle\langle i|,
$$

where $I=\sum_{i \in V}|i\rangle\langle i|$ is the identity. Indeed, in a SRG each vertex has degree $k$, so the diagonal degree matrix is $D=k I$ (see Figure 4). The basis states (23) are obtained, as follows:

$$
H\left|e_{1}\right\rangle=(k-i \kappa)\left|e_{1}\right\rangle-\sum_{(j, w) \in E}|j\rangle=(k-i \kappa)\left|e_{1}\right\rangle-\sqrt{k}\left|e_{2}\right\rangle \stackrel{\text { O.N. }}{\longrightarrow}\left|e_{2}\right\rangle .
$$

A remark is due in order to address the computation of the next basis states. The diameter of a connected SRG $G$, i.e., the maximum distance between two vertices of $G$, is 2 [62]. This means that, given a vertex $w$, we can group all the other vertices in two subsets, as follows: the subset of the vertices at a distance 1 from $w$ (adjacent); the subset of the vertices at a distance 2 from $w$ (nonadjacent). Because of the structure of the SRG, where two (non)adjacent vertices have $\lambda(\mu)$ common adjacent vertices, in the following we face summations with repeated terms.

To determine the third basis state, we consider

$$
H\left|e_{2}\right\rangle=k\left|e_{2}\right\rangle-\frac{1}{\sqrt{k}} \sum_{(i, w) \in E} \sum_{(j, i) \in E}|j\rangle=(k-\lambda)\left|e_{2}\right\rangle-\sqrt{k}\left|e_{1}\right\rangle-\sqrt{\mu(k-\lambda-1)}\left|e_{3}\right\rangle \stackrel{\text { O.N. }}{\longrightarrow}\left|e_{3}\right\rangle .
$$

To explain this, we have to focus on $\sum_{(i, w) \in E} \sum_{(j, i) \in E}|j\rangle$. The index of the first summation runs over the vertices $i$ adjacent to $w$, whereas the index of the second summation runs over the vertices $j$ adjacent to $i$. On the one hand, the vertex $w$ is counted $k$ times, because it has $k$ adjacent vertices $i$, each of which, in turn, has $j=w$ among its adjacent vertices. On the other hand, the index of the second summation runs over the vertices adjacent and nonadjacent to $w$, because of the structure of the SRG. Each vertex $j$ adjacent to $w$, i.e., $(j, w) \in E$, is connected to other $\lambda$ vertices adjacent to $w$, so it is counted $\lambda$ times. Each vertex $j$ nonadjacent to $w$, i.e., $(j, w) \notin E$, is connected to $\mu$ vertices adjacent to $w$, so it is counted $\mu$ times. Thus, we have

$$
\sum_{(i, w) \in E} \sum_{(j, i) \in E}|j\rangle=k\left|e_{1}\right\rangle+\lambda \sum_{(j, w) \in E}|j\rangle+\mu \sum_{(j, w) \notin E}|i\rangle=k\left|e_{1}\right\rangle+\lambda \sqrt{k}\left|e_{2}\right\rangle+\mu \sqrt{N-k-1}\left|e_{3}\right\rangle .
$$


Accordingly, according to Equation (22), we can write $\mu \sqrt{(N-k-1)}=\sqrt{\mu k(k-\lambda-1)}$, from which Equation (A10) follows.

Subsequently, we consider

$$
H\left|e_{3}\right\rangle=k\left|e_{3}\right\rangle-\frac{1}{\sqrt{N-k-1}} \sum_{(i, w) \notin E} \sum_{(j, i) \in E}|j\rangle=\mu\left|e_{3}\right\rangle-\sqrt{\mu(k-\lambda-1)}\left|e_{2}\right\rangle .
$$

Again, to explain this, we have to focus on the term $\sum_{(i, w) \notin E} \sum_{\left(j, i^{\prime}\right) \in E}|j\rangle$ in the second equality. The index of the first summation runs over the vertices $i$ nonadjacent to $w$, whereas the index of the second summation runs over the vertices $j$ adjacent to $i$. Each vertex $j$ nonadjacent to $w$, i.e., $(j, w) \notin E$, is connected to other $k-\mu$ vertices nonadjacent to $w$, so it is counted $k-\mu$ times. Each vertex $j$ adjacent to $w$, i.e., $(j, w) \in E$, is connected to $k-\lambda-1$ vertices nonadjacent to $w$, so it is counted $k-\lambda-1$ times. Thus, we have

$$
\begin{aligned}
\sum_{(i, w) \notin E} \sum_{(j, i) \in E}|j\rangle & =(k-\lambda-1) \sum_{(i, w) \in E}|i\rangle+(k-\mu) \sum_{(i, w) \notin E}|i\rangle \\
& =(k-\lambda-1) \sqrt{k}\left|e_{2}\right\rangle+(k-\mu) \sqrt{N-k-1}\left|e_{3}\right\rangle .
\end{aligned}
$$

So, according to Equation (22), we can write $(k-\lambda-1) \sqrt{k}=\sqrt{\mu(N-k-1)(k-\lambda-1)}$, from which Equation (A12) follows.

In conclusion, any state $H^{k}|w\rangle \in \operatorname{span}\left(\left\{\left|e_{1}\right\rangle,\left|e_{2}\right\rangle,\left|e_{3}\right\rangle\right\}\right) \forall k \in \mathbb{N}_{0}$, thus the states (23) form an orthonormal basis for the subspace $\mathcal{I}(H,|w\rangle)$.

Appendix B.3. Joined Complete Graphs

The Laplacian matrix of the two complete graphs $K_{N / 2}$ joined by a single edge $\left(b_{1}, b_{2}\right)$ is

$$
L=L_{1}+L_{2}+\underbrace{\left|b_{1}\right\rangle\left\langle b_{1}|+| b_{2}\right\rangle\left\langle b_{2}|-| b_{1}\right\rangle\left\langle b_{2}|-| b_{2}\right\rangle\left\langle b_{1}\right|}_{\text {bridge }},
$$

where

$$
L_{k}=\left(\frac{N}{2}-1\right) \sum_{i \in V_{k}}|i\rangle\left\langle i\left|-\sum_{(i, j) \in E_{k}}\right| i\right\rangle\langle j|
$$

is the Laplacian matrix of the complete graph $K_{N / 2}^{(k)}$, with $k=1,2$. The bridge introduces the edge between the vertices $b_{1}$ and $b_{2}$ and correctly makes the degree of such vertices be $N / 2$ (see Figure 5). Hence, $L|v\rangle=L_{k}|v\rangle$ for any vertex $v \in V_{k} \backslash\left\{b_{k}\right\}$. Instead, $L\left|b_{k}\right\rangle=$ $(N / 2)\left|b_{k}\right\rangle-\sum_{\left(i, b_{k}\right) \in E_{k}}|i\rangle-\left|b_{\bar{k}}\right\rangle$, where $\bar{k}$ is the complement of $k$ in $\{1,2\}$.

Reasoning by symmetry, we introduce the subsets of the identically evolving vertices, i.e., the subsets containing the vertices that behave identically under the action of the Hamiltonian:

$$
\begin{aligned}
H|w\rangle & =(N / 2-1-i \kappa)|w\rangle-\sum_{i \in V_{a}}|i\rangle-\left|b_{1}\right\rangle, \\
H \sum_{i \in V_{a}}|i\rangle & =2 \sum_{i \in V_{a}}|i\rangle-(N / 2-2)\left(|w\rangle+\left|b_{1}\right\rangle\right), \\
H\left|b_{1}\right\rangle & =N / 2\left|b_{1}\right\rangle-\sum_{i \in V_{a}}|i\rangle-|w\rangle-\left|b_{2}\right\rangle, \\
H\left|b_{2}\right\rangle & =N / 2\left|b_{2}\right\rangle-\sum_{i \in V_{c}}|i\rangle-\left|b_{1}\right\rangle, \\
H \sum_{i \in V_{c}}|i\rangle & =\sum_{i \in V_{c}}|i\rangle-(N / 2-1)\left|b_{2}\right\rangle,
\end{aligned}
$$

where $V_{a}=V_{1} \backslash\left\{w, b_{1}\right\}$ and $V_{c}=V_{2} \backslash\left\{b_{2}\right\}$. Note that the results of $H$ applied on the vertices $b_{1}$ or $b_{2}$ are different, and this is the reason why they form different subsets. According to these preliminary results, the basis states (28) are obtained, as follows: 


$$
\begin{aligned}
H\left|e_{1}\right\rangle= & (N / 2-1-i \kappa)|w\rangle-\sum_{i \in V_{a}}|i\rangle-\left|b_{1}\right\rangle \stackrel{\text { O.N. }}{\longrightarrow}\left|e_{2}\right\rangle, \\
H\left|e_{2}\right\rangle= & \frac{1}{\sqrt{N / 2-1}}\left[-(N / 2-1)|w\rangle+\sum_{i \in V_{a}}|i\rangle+2\left|b_{1}\right\rangle-\left|b_{2}\right\rangle\right] \stackrel{\text { O.N. }}{\longrightarrow}\left|e_{3}\right\rangle, \\
H\left|e_{3}\right\rangle= & \frac{1}{\sqrt{(N-3)(N / 2-1)}}\left[N / 2 \sum_{i \in V_{a}}|i\rangle-\left(N^{2} / 4-3\right)\left|b_{1}\right\rangle+\left(N^{2} / 4-2\right)\left|b_{2}\right\rangle\right. \\
& \left.-(N / 2-1) \sum_{i \in V_{c}}|i\rangle\right] \stackrel{\text { O.N. }}{\longrightarrow}\left|e_{4}\right\rangle,
\end{aligned}
$$

and it can be proved that

$$
H\left|e_{4}\right\rangle=\frac{\sqrt{N / 2-1}}{N-3}\left(\sqrt{N(N / 2-2)+1}\left|e_{3}\right\rangle+\sqrt{N / 2-1}\left|e_{4}\right\rangle\right) .
$$

In conclusion, any state $H^{k}|w\rangle \in \operatorname{span}\left(\left\{\left|e_{1}\right\rangle, \ldots,\left|e_{4}\right\rangle\right\}\right) \forall k \in \mathbb{N}_{0}$, thus the states (28) form an orthonormal basis for the subspace $\mathcal{I}(H,|w\rangle)$.

\section{Appendix B.4. Simplex of Complete Graphs}

The Laplacian matrix is defined as $L=D-A$. For a $M$-simplex of complete graphs the diagonal degree matrix is $D=M I$, since the graph is regular, and the adjacency matrix is $A=\sum_{m=1}^{M+1} A_{\text {intra }}^{(m)}+A_{\text {inter, }}$ where

$$
A_{\text {intra }}^{(m)}=\sum_{(i, j) \in E_{m}}\left|i^{(m)}\right\rangle\left\langle j^{(m)}\right|
$$

is the intra-graph adjacency matrix, i.e., within the complete graph $K_{M}^{(m)}$, and

$$
A_{\text {inter }}=\sum_{m=1}^{M+1} \sum_{i=1}^{M}\left|i^{(m)}\right\rangle\left\langle(M+1-i)^{\left(m^{\prime}\right)}\right|,
$$

with $m^{\prime}=1+\bmod (i+m-1, M+1)$, is the inter-graphs adjacency matrix, i.e., between different complete graphs. The index $m$ labels the complete graphs $K_{M}^{(m)}$ forming the $M$ simplex. Note that Equation (A26) follows the labeling of the vertices in Figure A1 and it is just one of the possible ways to computationally implement the inter-graphs contribution.

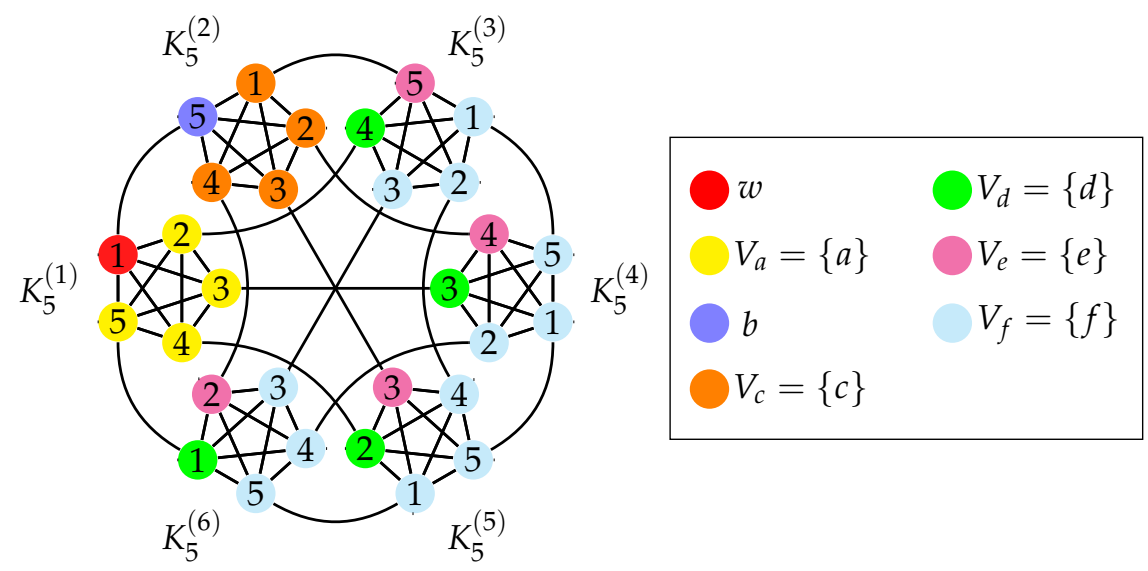

Figure A1. Labeling of vertices in a 5-simplex of complete graphs. The trap vertex $w$ is colored red and assumed to be $|1\rangle$ in $K_{5}^{(1)}$. Same coloring denotes the subsets $V_{\alpha}$ of identically evolving vertices $\alpha$, with $\alpha=w, a, b, c, d, e, f$ (see also Figure 6). Note that each of the two vertices $w$ and $b$ forms a subset of one element, itself. 
In this case, using the notion of adjacency and reasoning by symmetry to introduce the subsets of the identically evolving vertices provide a framework which, analytically, is simpler and clearer to deal with than explicitly using the Laplacian above defined. These subsets contain the vertices which behave identically under the action of the Hamiltonian:

$$
\begin{aligned}
H|w\rangle & =(M-i \kappa)|w\rangle-\sum_{i \in V_{a}}|i\rangle-|b\rangle, \\
H \sum_{i \in V_{a}}|i\rangle & =2 \sum_{i \in V_{a}}|i\rangle-(M-1)|w\rangle-\sum_{i \in V_{d}}|i\rangle, \\
H|b\rangle & =M|b\rangle-|w\rangle-\sum_{i \in V_{c}}|i\rangle, \\
H \sum_{i \in V_{c}}|i\rangle & =2 \sum_{i \in V_{c}}|i\rangle-(M-1)|b\rangle-\sum_{i \in V_{e}}|i\rangle, \\
H \sum_{i \in V_{d}}|i\rangle & =M \sum_{i \in V_{d}}|i\rangle-\sum_{i \in V_{a}}|i\rangle-\sum_{i \in V_{e}}|i\rangle-\sum_{i \in V_{f}}|i\rangle, \\
H \sum_{i \in V_{e}}|i\rangle & =M \sum_{i \in V_{e}}|i\rangle-\sum_{i \in V_{c}}|i\rangle-\sum_{i \in V_{d}}|i\rangle-\sum_{i \in V_{f}}|i\rangle, \\
H \sum_{i \in V_{f}}|i\rangle & =2 \sum_{i \in V_{f}}|i\rangle-(M-2)\left(\sum_{i \in V_{d}}|i\rangle+\sum_{i \in V_{e}}|i\rangle\right) .
\end{aligned}
$$

Note that the results of $H$ applied on the vertices in $V_{c}$ or in $V_{d}$ are different, and this is the reason why they form different subsets. According to these preliminary results, the basis states (33) are obtained, as follows:

$$
\begin{aligned}
H\left|e_{1}\right\rangle= & (M-i \kappa)|w\rangle-\sum_{i \in V_{a}}|i\rangle-|b\rangle \stackrel{\text { O.N. }}{\longrightarrow}\left|e_{2}\right\rangle, \\
H\left|e_{2}\right\rangle= & \frac{1}{\sqrt{M}}\left(2 \sum_{i \in V_{a}}|i\rangle-M|w\rangle+M|b\rangle-\sum_{i \in V_{c} \cup V_{d}}|i\rangle\right) \stackrel{\text { O.N. }}{\longrightarrow}\left|e_{3}\right\rangle, \\
H\left|e_{3}\right\rangle= & \frac{\sqrt{M}}{\sqrt{(M-1)\left(M^{2}-2 M+4\right)}}\left[\frac{M-4}{M} \sum_{i \in V_{a}}|i\rangle-(M-1)^{2}|b\rangle\right. \\
& \left.+\frac{M^{2}-M+2}{M} \sum_{i \in V_{c} \cup V_{d}}|i\rangle-2 \sum_{i \in V_{e}}|i\rangle-\sum_{i \in V_{f}}|i\rangle\right] \stackrel{\text { O.N. }}{\longrightarrow}\left|e_{4}\right\rangle, \\
H\left|e_{4}\right\rangle= & \frac{1}{\sqrt{(M-1)\left(M^{2}-2 M+4\right)\left(M^{3}+2 M^{2}-8 M+16\right)}}\left\{\left(M^{2}-4\right)\left[\sum_{i \in V_{a}}|i\rangle-(M-1)|b\rangle\right]\right. \\
& \left.+2\left(M^{2}-M+2\right) \sum_{i \in V_{c} \cup V_{d}}|i\rangle-M\left(M^{2}-2 M+8\right) \sum_{i \in V_{e}}|i\rangle+(M-2)^{2} \sum_{i \in V_{f}}|i\rangle\right\} \stackrel{\text { O.N. }}{\longrightarrow}\left|e_{5}\right\rangle,
\end{aligned}
$$

and it can be proved that

$$
H\left|e_{5}\right\rangle=\frac{M+2}{M^{3}+2 M^{2}-8 M+16}\left[M \sqrt{(M-2)\left(M^{2}-2 M+4\right)}\left|e_{4}\right\rangle+\left(M^{3}-4 M+8\right)\left|e_{5}\right\rangle\right] .
$$

In conclusion, any state $H^{k}|w\rangle \in \operatorname{span}\left(\left\{\left|e_{1}\right\rangle, \ldots,\left|e_{5}\right\rangle\right\}\right) \forall k \in \mathbb{N}_{0}$, thus the states (33) form an orthonormal basis for the subspace $\mathcal{I}(H,|w\rangle)$.

\section{References}

1. Farhi, E.; Gutmann, S. Quantum computation and decision trees. Phys. Rev. A 1998, 58, 915. [CrossRef]

2. Childs, A.M.; Farhi, E.; Gutmann, S. An example of the difference between quantum and classical random walks. Quantum Inf. Process. 2002, 1, 35-43. [CrossRef]

3. Wang, J.; Manouchehri, K. Physical Implementation of Quantum Walks; Springer: New York, NY, USA, 2013.

4. $\mathrm{Du}$, J.; Li, H.; Xu, X.; Shi, M.; Wu, J.; Zhou, X.; Han, R. Experimental implementation of the quantum random-walk algorithm. Phys. Rev. A 2003, 67, 042316. [CrossRef] 
5. Côté, R.; Russell, A.; Eyler, E.E.; Gould, P.L. Quantum random walk with Rydberg atoms in an optical lattice. New J. Phys. 2006, 8, 156. [CrossRef]

6. Qiang, X.; Loke, T.; Montanaro, A.; Aungskunsiri, K.; Zhou, X.; O’Brien, J.L.; Wang, J.B.; Matthews, J.C. Efficient quantum walk on a quantum processor. Nat. Commun. 2016, 7,1-6.

7. Tang, H.; Lin, X.F.; Feng, Z.; Chen, J.Y.; Gao, J.; Sun, K.; Wang, C.Y.; Lai, P.C.; Xu, X.Y.; Wang, Y.; et al. Experimental two-dimensional quantum walk on a photonic chip. Sci. Adv. 2018, 4, eaat3174. [CrossRef]

8. Venegas-Andraca, S.E. Quantum Walks for Computer Scientists; Synthesis Lectures on Quantum Computing; Morgan \& Claypool Publishers: San Rafael, CA, USA, 2008.

9. Portugal, R. Quantum Walks and Search Algorithms; Springer: Berlin/Heidelberg, Germany, 2018.

10. Childs, A.M.; Goldstone, J. Spatial search by quantum walk. Phys. Rev. A 2004, 70, 022314. [CrossRef]

11. Krok, M.; Rycerz, K.; Bubak, M. Application of Continuous Time Quantum Walks to Image Segmentation. In International Conference on Computational Science; Springer: Cham, Switzerland, 2019, pp. 17-30.

12. Lahini, Y.; Steinbrecher, G.R.; Bookatz, A.D.; Englund, D. Quantum logic using correlated one-dimensional quantum walks. Npj Quantum Inf. 2018, 4, 2. [CrossRef]

13. Childs, A.M. Universal computation by quantum walk. Phys. Rev. Lett. 2009, 102, 180501. [CrossRef]

14. Christandl, M.; Datta, N.; Ekert, A.; Landahl, A.J. Perfect state transfer in quantum spin networks. Phys. Rev. Lett. 2004, 92, 187902. [CrossRef]

15. Kendon, V.M.; Tamon, C. Perfect state transfer in quantum walks on graphs. J. Comput. Theor. Nanosci. 2011, 8, 422-433. [CrossRef]

16. Alvir, R.; Dever, S.; Lovitz, B.; Myer, J.; Tamon, C.; Xu, Y.; Zhan, H. Perfect state transfer in Laplacian quantum walk. J. Algebr. Comb. 2016, 43, 801-826. [CrossRef]

17. Lahini, Y.; Verbin, M.; Huber, S.D.; Bromberg, Y.; Pugatch, R.; Silberberg, Y. Quantum walk of two interacting bosons. Phys. Rev. A 2012, 86, 011603. [CrossRef]

18. Beggi, A.; Razzoli, L.; Bordone, P.; Paris, M.G.A. Probing the sign of the Hubbard interaction by two-particle quantum walks. Phys. Rev. A 2018, 97, 013610. [CrossRef]

19. Agliari, E.; Blumen, A.; Mülken, O. Dynamics of continuous-time quantum walks in restricted geometries. J. Phys. A 2008, 41, 445301. [CrossRef]

20. Salimi, S. Continuous-time quantum walks on semi-regular spidernet graphs via quantum probability theory. Quantum Inf. Process. 2010, 9, 75-91. [CrossRef]

21. Darázs, Z.; Anishchenko, A.; Kiss, T.; Blumen, A.; Mülken, O. Transport properties of continuous-time quantum walks on Sierpinski fractals. Phys. Rev. E 2014, 90, 032113. [CrossRef]

22. Li, X.; Chen, H.; Wu, M.; Ruan, Y.; Liu, Z.; Tan, J. Quantum transport on large-scale sparse regular networks by using continuous-time quantum walk. Quantum Inf. Process. 2020, 19, 1-13. [CrossRef]

23. Rai, A.; Agarwal, G.S.; Perk, J.H. Transport and quantum walk of nonclassical light in coupled waveguides. Phys. Rev. A 2008, 78, 042304. [CrossRef]

24. Blumen, A.; Bierbaum, V.; Mülken, O. Coherent dynamics on hierarchical systems. Phys. A 2006, 371, 10-15. [CrossRef]

25. Mülken, O.; Pernice, V.; Blumen, A. Quantum transport on small-world networks: A continuous-time quantum walk approach. Phys. Rev. E 2007, 76, 051125. [CrossRef] [PubMed]

26. Xu, X.P.; Li, W.; Liu, F. Coherent transport on Apollonian networks and continuous-time quantum walks. Phys. Rev. E 2008, 78, 052103. [CrossRef] [PubMed]

27. Yalouz, S.; Pouthier, V. Continuous-time quantum walk on an extended star graph: Trapping and superradiance transition. Phys. Rev. E 2018, 97, 022304. [CrossRef] [PubMed]

28. Mülken, O.; Blumen, A. Continuous-time quantum walks: Models for coherent transport on complex networks. Phys. Rep. 2011, 502, 37-87. [CrossRef]

29. Mülken, O.; Blumen, A.; Amthor, T.; Giese, C.; Reetz-Lamour, M.; Weidemüller, M. Survival probabilities in coherent exciton transfer with trapping. Phys. Rev. Lett. 2007, 99, 090601. [CrossRef] [PubMed]

30. Agliari, E.; Muelken, O.; Blumen, A. Continuous-time quantum walks and trapping. Int. J. Bifurc. Chaos 2010, 20, 271-279. [CrossRef]

31. Mülken, O.; Blumen, A. Slow transport by continuous time quantum walks. Phys. Rev. E 2005, 71, 016101. [CrossRef]

32. Mülken, O.; Blumen, A. Efficiency of quantum and classical transport on graphs. Phys. Rev. E 2006, 73, 066117. [CrossRef]

33. Lambert, N.; Chen, Y.N.; Cheng, Y.C.; Li, C.M.; Chen, G.Y.; Nori, F. Quantum biology. Nat. Phys. 2013, 9, 10-18. [CrossRef]

34. Mohseni, M.; Omar, Y.; Engel, G.S.; Plenio, M.B. Quantum Effects in Biology; Cambridge University Press: Cambridge, UK, 2014.

35. Mülken, O.; Bierbaum, V.; Blumen, A. Coherent exciton transport in dendrimers and continuous-time quantum walks. J. Chem. Phys. 2006, 124, 124905. [CrossRef]

36. Mohseni, M.; Rebentrost, P.; Lloyd, S.; Aspuru-Guzik, A. Environment-assisted quantum walks in photosynthetic energy transfer. J. Chem. Phys. 2008, 129, 174106. [CrossRef] [PubMed]

37. Rebentrost, P.; Mohseni, M.; Kassal, I.; Lloyd, S.; Aspuru-Guzik, A. Environment-assisted quantum transport. New J. Phys. 2009, 11, 033003. [CrossRef] 
38. Plenio, M.B.; Huelga, S.F. Dephasing-assisted transport: Quantum networks and biomolecules. New J. Phys. $2008,10,113019$. [CrossRef]

39. Olaya-Castro, A.; Lee, C.F.; Olsen, F.F.; Johnson, N.F. Efficiency of energy transfer in a light-harvesting system under quantum coherence. Phys. Rev. B 2008, 78, 085115. [CrossRef]

40. Caruso, F.; Chin, A.W.; Datta, A.; Huelga, S.F.; Plenio, M.B. Highly efficient energy excitation transfer in light-harvesting complexes: The fundamental role of noise-assisted transport. J. Chem. Phys. 2009, 131, 09B612. [CrossRef]

41. Hoyer, S.; Sarovar, M.; Whaley, K.B. Limits of quantum speedup in photosynthetic light harvesting. New J. Phys. 2010, $12,065041$. [CrossRef]

42. Novo, L.; Mohseni, M.; Omar, Y. Disorder-assisted quantum transport in suboptimal decoherence regimes. Sci. Rep. 2016, 6, 18142. [CrossRef]

43. Adronov, A.; Fréchet, J.M. Light-harvesting dendrimers. Chem. Commun. 2000, 18, 1701-1710.

44. Bradshaw, D.S.; Andrews, D.L. Mechanisms of light energy harvesting in dendrimers and hyperbranched polymers. Polymers 2011, 3, 2053-2077. [CrossRef]

45. Wong, T.G.; Tarrataca, L.; Nahimov, N. Laplacian versus adjacency matrix in quantum walk search. Quantum Inf. Process. 2016, 15, 4029-4048. [CrossRef]

46. Janmark, J.; Meyer, D.A.; Wong, T.G. Global symmetry is unnecessary for fast quantum search. Phys. Rev. Lett. 2014, 112, 210502. [CrossRef]

47. Meyer, D.A.; Wong, T.G. Connectivity is a poor indicator of fast quantum search. Phys. Rev. Lett. 2015, 114, 110503. [CrossRef] [PubMed]

48. Novo, L.; Chakraborty, S.; Mohseni, M.; Neven, H.; Omar, Y. Systematic dimensionality reduction for quantum walks: Optimal spatial search and transport on non-regular graphs. Sci. Rep. 2015, 5, 13304. [CrossRef] [PubMed]

49. Jafarizadeh, M.; Sufiani, R.; Salimi, S.; Jafarizadeh, S. Investigation of continuous-time quantum walk by using Krylov subspace-Lanczos algorithm. Eur. Phys. J. B 2007, 59, 199-216. [CrossRef]

50. Wong, T.G. Diagrammatic approach to quantum search. Quantum Inf. Process. 2015, 14, 1767-1775. [CrossRef]

51. Cameron, P.J.; Van Lint, J.H.; Cameron, P.J. Designs, Graphs, Codes and Their Links; Cambridge University Press: Cambridge, UK, 1991; Volume 3.

52. Brouwer, A.E.; Haemers, W.H. Spectra of Graphs; Springer Science \& Business Media: Berlin/Heidelberg, Germany, 2011.

53. Weisstein, E.W. Prime Power. From MathWorld-A Wolfram Web Resource. Available online: https://mathworld.wolfram. com/PrimePower.html (accessed on 20 October 2020).

54. Nelson, D.R.; Fisher, M.E. Soluble renormalization groups and scaling fields for low-dimensional Ising systems. Ann. Phys. 1975, 91, 226-274. [CrossRef]

55. Dhar, D. Lattices of effectively nonintegral dimensionality. J. Math. Phys. 1977, 18, 577-585. [CrossRef]

56. Dhar, D. Self-avoiding random walks: Some exactly soluble cases. J. Math. Phys. 1978, 19, 5-11. [CrossRef]

57. Wang, Y.; Wu, S.; Wang, W. Optimal quantum search on truncated simplex lattices. Phys. Rev. A 2020, 101, 062333. [CrossRef]

58. Fiedler, M. Laplacian of graphs and algebraic connectivity. Banach Cent. Publ. 1989, 25, 57-70. [CrossRef]

59. West, D.B. Introduction to Graph Theory, 2nd ed.; Prentice Hall: Upper Saddle River, NJ, USA, 2001.

60. Fiedler, M. Algebraic connectivity of graphs. Czechoslov. Math. J. 1973, 23, 298-305. [CrossRef]

61. De Abreu, N.M.M. Old and new results on algebraic connectivity of graphs. Linear Algebra Its Appl. 2007, 423, 53-73. [CrossRef]

62. Beineke, L.W.; Wilson, R.J.; Cameron, P.J. (Eds.) Topics in Algebraic Graph Theory; Cambridge University Press: Cambridge, UK, 2004; Volume 102.

63. Chartrand, G.; Zhang, P. A First Course in Graph Theory; Dover Publications: Mineola, NY, USA, 2012.

64. Chung, F.R.; Graham, F.C. Spectral Graph Theory; Number 92; American Mathematical Soc.: Providence, RI, USA, 1997.

65. Banerjee, S.; Roy, A. Linear Algebra and Matrix Analysis for Statistics; CRC Press: Boca Raton, FL, USA, 2014. 